\title{
PLANEAMIENTO ESTRATÉGICO DE TECNOLOGÍAS DE INFORMACIÓN PARA LA UNIDAD DE POSGRADO DE LA FACULTAD DE INGENIERÍA MECÁNICA DE LA UNCP
}

\section{TRATEGIC PLANNING OF INFORMATION TECHNOLOGIES FOR THE POSTGRADUATE UNIT OF THE FACULTY OF MECHANICAL ENGINEERING OF THE UNCPPAPERSON}

\author{
Dr. Alex Sandro Landeo Q*, Mag. Miguel Oracio Camarena I** \\ "Escuela de Posgrado, Universidad Peruana Los Andes \\ Av. Giráldez No 230 Huancayo, Junín, Perú \\ Teléfono y Fax: 51-064 224479 \\ d.alandeo@upla.edu.pe \\ **Facultad de Ingeniería de Sistemas, Universidad Nacional del Centro del Perú \\ Av. Mariscal Castilla N 3909 - El Tambo - Huancayo, Junín, Perú \\ Teléfono y Fax: 51-064481060 \\ mcamarena@uncp.edu.pe.
}

Resumen: La Investigación tuvo como problema: ¿En qué medida el Planeamiento Estratégico de Tecnologías de Información influye en la ventaja competitiva de la Unidad de Posgrado de la Facultad de Ingeniería Mecánica de la UNCP?; siendo el Objetivo: Determinar en qué medida el Planeamiento Estratégico de Tecnologías de Información influye en la ventaja competitiva de la Unidad de Posgrado de la Facultad de Ingeniería Mecánica de la UNCP. El tipo de investigación fue el aplicado, el nivel explicativo y con un diseño no experimental transeccional. Los métodos utilizados fueron: analítico-sintético y de forma particular el método de la Ingeniería de Sistemas se tuvo una muestra dirigida (No Probabilística) y un muestreo aleatorio simple. Los instrumentos de recolección de Información utilizados fueron la ficha de observación y análisis documental. Concluyéndose que la implementación del Plan Estratégico de Tecnología de Información (PETI) en la UPG de la Facultad de Ingeniería Mecánica de la UNCP, implica cambios significativos en las estrategias de negocio tanto a nivel operativo y de Tecnologías de Información (TI), los mismo que se materializan con la implementación de laboratorios especializados de mecánica que incluye el uso de simuladores, renovación de laboratorios de cómputo, implementación de nuevos sistemas de información, rediseño de los procesos estratégicos y operativos. Aspectos estos que permitirán a la organización una gestión estratégica de la información.

Palabras clave: Planeamiento Estratégico de Tecnologías de Información, ventaja competitiva, Unidad de Posgrado, Facultad de Ingeniería Mecánica

\begin{abstract}
The research had as problem: To what extent does the Strategic Planning of Information Technologies influence the competitive advantage of the Postgraduate Unit of the Faculty of Mechanical Engineering of the UNCP?; being the Objective: To determine to what extent the Strategic Planning of Information Technologies influences the competitive advantage of the Postgraduate Unit of the Faculty of Mechanical Engineering of the UNCP. The type of research was applied, the explanatory level and with a nonexperimental transectional design. The methods used were: analytical-synthetic and, in particular, the System Engineering method, a directed sample (Non-Probabilistic) and a simple random sampling were taken. The information collection instruments used were the observation and documentary analysis sheet. Concluding that the implementation of the Strategic Information Technology Plan (PETI) in the UPG of the Faculty of Mechanical Engineering of the UNCP, implies significant changes in business strategies both at the operational level and Information Technology (IT), the same that materialize with the implementation of specialized laboratories of mechanics that includes the use of simulators, renewal of computer laboratories, implementation of new information systems, redesign of
\end{abstract}


strategic and operational processes. These aspects that will allow the organization a strategic information management.

Keywords: Strategic Information Technology Planning, competitive advantage, Postgraduate Unit, Faculty of Mechanical Engineering.

\section{INTRODUCCIÓN}

Las organizaciones en la actualidad se encuentran en un momento crucial y decisivo en cuanto a la implementación y uso de la tecnología, que le permita fortalecer y consolidar su potencial humano; aspecto este que está marcado por la globalización del internet, cuya masificación y cambio acelerado producen que las interrelaciones sociales, comerciales, políticas y personales cambien de manera rápida y de forma continua a fin de ir al compás con la evolución de las tecnologías de información. Para ello se necesita que en las organizaciones los especialistas en TI sean innovadores en cuanto al uso del internet, debiéndolo considerar como el punto de partida para confluir esfuerzos creativos que permitan diseñar y proponer nuevos productos y servicios aprovechando las oportunidades de la red para satisfacer plenamente los requerimientos de los clientes

Del mismo modo se debe considerar que actualmente la competitividad empresarial está a un nivel altísimo, toda vez que éstas han generalizado el uso de las tecnologías; pero se debe comprender que este uso no debe ser de forma improvisada ni mucho menos al azar, por el contrario debe ser debidamente planificada y organizada a fin de evitar inversiones innecesarias e improductivas. Por lo tanto las organizaciones están en la obligación de identificar concienzudamente su potencial tecnológico y sobre todo las recientes tendencias y requerimientos de Tecnologías de Información que le permitan establecer ventajas competitivas en la gestión de su información; para lo cual un PETI, es necesario a fin de alinear las propuestas y alternativas de TI a las estrategias de la empresa, esto con la finalidad de asegurar que la adopción de las tecnologías sean el soporte para los procesos de negocio y contribuyan con efectividad al desarrollo y evolución de la organización mediante sus ventajas competitivas.

\section{MATERIALES Y MÉTODOS}

Materiales: El tipo de investigación fue el aplicado y del nivel explicativo, empleando un diseño no experimental de corte transversal. Se empleó la observación y el análisis documental cómo técnicas de recolección de datos. La muestra de investigación fue una muestra dirigida (No Probabilística).

Métodos: Análisis Síntesis- que permitió realizar un estudio del Planeamiento Estratégico de Tecnologías de Información y sus elementos a fin de comprender su naturaleza y alcance. Cómo método particular se empleó el Método de la Ingeniería de Sistemas, que permitió abordar y resolver la problemática mediante un modelo del sistema y sus especificaciones; mediante éste se recogió, organizó, analizó e interpretó los datos pertinentes de los postulados del Planeamiento Estratégico de Tecnologías de
Información para determinar el modelo para la organización bajo estudio.

\section{RESULTADOS}

1. Modelamiento Estratégico a Nivel Corporativo de la Universidad Nacional Del Centro Del Perú.

"La Universidad Nacional del Centro del Perú se creó el 16 de diciembre de 1959, mediante el Decreto Supremo $\mathrm{N}^{\circ} 46$ iniciando sus actividades el 3 de abril de 1960 con 14 facultades, siendo su primer rector el Dr. Javier Pulgar Vidal, comenzando sus actividades" (UNCP, 1999).

\section{Facultades}

Actualmente la UNCP cuenta con 22 facultades en la sede principal y 3 carreras profesionales en las sedes de Satipo, Tarma, Junín

\section{Productos y/o Servicios y Clientes}

- Productos: La Universidad Nacional del Centro del Perú a través de la Facultad de Ingeniería de Industria Alimentarias ofrece a toda la comunidad universitaria productos lácteos como son: yogurth, helados, queso, mantequilla entre otros. El local de expendio es en la misma universidad, la administración producción y venta está a cargo de la misma facultad.

- Servicios: Los servicios ofrecidos en la UNCP son: de educación y asesoría (Educación, Asesoría en producción agropecuaria y Asesoría en análisis y procesamiento de minerales).

- Clientes: Para los productos lácteos, son todos los integrantes de la comunidad universitaria. En cuanto a los servicios de asesoría en producción agropecuaria los clientes son los agricultores del valle del Mantaro. Los clientes del servicio de análisis de minerales son las empresas mineras de la región. Los clientes del servicio de educación son todos los estudiantes de los diferentes niveles académicos que ofrece la UNCP como: Pre Universitario, idiomas, pregrado y posgrado.

\section{Cartera de Negocios}

Las UENs que brindan productos y servicio en la UNCP son:

- Productos lácteos.

- Educación

- Asesoría en producción agropecuaria.

- Asesoría en análisis y procesamiento de minerales. 


\section{Matriz de Priorización de UENs}

\begin{tabular}{|c|c|c|c|c|c|c|c|c|c|}
\hline \multirow{2}{*}{$\begin{array}{l}\text { CRITERIO DE } \\
\text { PRIORIZACION } \\
\text { UEN }\end{array}$} & 0,2 & 0,1 & 0,1 & 0,1 & 0,2 & 0,1 & 0,2 & 1 & \multirow[b]{2}{*}{ 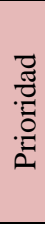 } \\
\hline & $\begin{array}{l}\frac{0}{0} \\
\frac{10}{0} \\
\infty \\
\infty\end{array}$ & 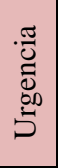 & 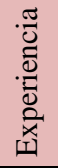 & $\begin{array}{l}\frac{0}{0} \\
\frac{0}{0} \\
0\end{array}$ & 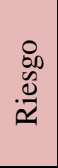 & 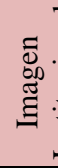 & 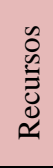 & $\begin{array}{c}\stackrel{\Xi}{\Xi} \\
\Xi \\
\tilde{\Xi}\end{array}$ & \\
\hline Productos lácteos & 0,4 & 0,2 & 0,4 & 0,3 & 0,6 & 0,4 & 0,8 & 3,1 & 4 \\
\hline Educación & 0,8 & 0,4 & 0,5 & 0,5 & 0,8 & 0,3 & 0,8 & 4,1 & 1 \\
\hline $\begin{array}{l}\text { Centro de } \\
\text { producción } \\
\text { agrícola }\end{array}$ & 0,6 & 0,3 & 0,2 & 0,4 & 0,8 & 0,3 & 0,8 & 3,4 & 2 \\
\hline $\begin{array}{l}\text { Centro de } \\
\text { producción } \\
\text { metalúrgica }\end{array}$ & 0,6 & 0,4 & 0,5 & 0,4 & 0,4 & 0,3 & 0,6 & 3,2 & 3 \\
\hline
\end{tabular}

Para determinar los valores de la tabla se ha multiplicado el peso ponderado por el valor del criterio, teniendo en consideración lo siguiente:

Peso ponderado:

- Beneficio

- Urgencia

01

- Experiencia

01

- Presión

0.1

- Riesgo

- Imagen Institucional:

- Recursos

Valor del Criterio:

- Muy alta : 5

- Alta : 4

- Mediana : 3

- Baja : 2

- Muy baja : : 1

Utilizando los mismos ponderados y los mismos valores de criterio se hizo la priorización en el servicio educación.

\section{Priorización por el servicio educación}

\begin{tabular}{|c|c|c|c|c|c|c|c|c|c|}
\hline CRITERIO DE & 0,2 & 0,1 & 0,1 & 0,1 & 0,2 & 0,1 & 0,2 & 1 & \\
\hline PRIORIZACION & $\begin{array}{l}\stackrel{0}{0} \\
\frac{0}{0} \\
\frac{0}{0} \\
\infty\end{array}$ & $\begin{array}{l}\cdot \frac{\pi}{0} \\
5 \\
5 \\
5 \\
5\end{array}$ & $\begin{array}{l}\frac{\pi}{0} \\
\frac{0}{0} \\
\frac{0}{0} \\
\frac{0}{x} \\
\text { I. }\end{array}$ & 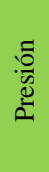 & 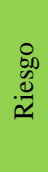 & 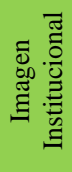 & 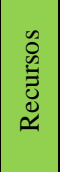 & $\stackrel{\tilde{E}}{\Xi}$ & $\begin{array}{l}\frac{\pi}{\pi} \\
\frac{\pi}{0} \\
0 \\
0\end{array}$ \\
\hline POSGRADO & 0,8 & 0,4 & 0,5 & 0,5 & 0,8 & 0,3 & 0,8 & 4,1 & 1 \\
\hline Pre Grado & 0,4 & 0,2 & 0,4 & 0,3 & 0,6 & 0,4 & 0,8 & 3,1 & 4 \\
\hline $\begin{array}{l}\text { Centro de } \\
\text { Idiomas }\end{array}$ & 0,6 & 0,3 & 0,2 & 0,4 & 0,8 & 0,3 & 0,8 & 3,4 & 2 \\
\hline Centro Pre & 0,6 & 0,4 & 0,5 & 0,4 & 0,4 & 0,3 & 0,6 & 3,2 & 3 \\
\hline
\end{tabular}

\section{Priorización por unidades de posgrado}

\begin{tabular}{|c|c|c|c|c|c|c|c|c|c|}
\hline CRITERIO DE & 0,2 & 0,1 & 0,1 & 0,1 & 0,2 & 0,1 & 0,2 & 1 & \\
\hline PRIORIZACION & $\begin{array}{l}\overline{\vec{U}} \\
\overline{\vec{J}} \\
\overline{0}\end{array}$ & $\begin{array}{l}\frac{\pi}{2} \\
0 \\
0 \\
5 \\
5\end{array}$ & $\frac{\mathbb{E}}{\frac{0}{0}}$ & 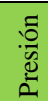 & 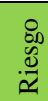 & $\begin{array}{l}\tilde{D} \\
\mathbb{D} \\
\stackrel{\Xi}{\Xi} \\
\end{array}$ & 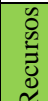 & $\begin{array}{l}\tilde{G} \\
\text { ज }\end{array}$ & $\frac{0}{2}$ \\
\hline
\end{tabular}

\begin{tabular}{|l|c|c|c|c|c|c|c|c|c|}
\multicolumn{1}{l|}{} & & & & & & & & & \\
UEN & & & & & & & & & \\
\hline $\begin{array}{l}\text { UPG } \\
\text { ADMINISTRACION }\end{array}$ & 0,4 & 0,3 & 0,4 & 0,3 & 0,7 & 0,4 & 0,8 & 3,3 & 8 \\
\hline $\begin{array}{l}\text { UPG DE } \\
\text { CONTABILIDAD }\end{array}$ & 0,6 & 0,3 & 0,2 & 0,4 & 0,8 & 0,3 & 0,8 & 3,4 & 7 \\
\hline $\begin{array}{l}\text { UPG DE ING. } \\
\text { MECANICA }\end{array}$ & 0,8 & 0,4 & 0,5 & 0,5 & 0,8 & 0,3 & 0,8 & 4,1 & 1 \\
\hline $\begin{array}{l}\text { UPG DE ING. } \\
\text { SISTEMAS }\end{array}$ & 0,4 & 0,2 & 0,4 & 0,3 & 0,7 & 0,4 & 0,8 & 3,2 & 9 \\
\hline UPG DE ING. MINAS & 0,6 & 0,4 & 0,4 & 0,4 & 0,8 & 0,3 & 0,8 & 3,7 & 4 \\
\hline $\begin{array}{l}\text { UPG DE ING. } \\
\text { QUIMICA }\end{array}$ & 0,6 & 0,4 & 0,5 & 0,3 & 0,4 & 0,3 & 0,6 & 3,1 & 10 \\
\hline $\begin{array}{l}\text { UPG DE ING. } \\
\text { ELECTRICA }\end{array}$ & 0,4 & 0,4 & 0,4 & 0,5 & 0,6 & 0,4 & 0,8 & 3,5 & 6 \\
\hline $\begin{array}{l}\text { UPG DE } \\
\text { INDUSTRIAS ALIM }\end{array}$ & 0,6 & 0,3 & 0,4 & 0,4 & 0,8 & 0,3 & 0,8 & 3,6 & 5 \\
\hline UPG DE PEDAGOGIA & 0,6 & 0,4 & 0,3 & 0,4 & 0,4 & 0,3 & 0,6 & 3,0 & 11 \\
\hline $\begin{array}{l}\text { UPG DE } \\
\text { FORESTALES }\end{array}$ & 0,4 & 0,2 & 0,2 & 0,3 & 0,6 & 0,4 & 0,8 & 2,9 & 12 \\
\hline \begin{tabular}{l} 
UPG DE MEDICINA \\
\hline UPG DE ECONOMIA
\end{tabular} & 0,6 & 0,4 & 0,4 & 0,4 & 0,8 & 0,4 & 0,8 & 3,8 & 3 \\
\hline $\begin{array}{l}\text { UPG DE } \\
\text { ARQUITECTURA }\end{array}$ & 0,4 & 0,2 & 0,3 & 0,4 & 0,4 & 0,3 & 0,6 & 2,8 & 13 \\
\hline $\begin{array}{l}\text { UPG DE } \\
\text { AGRONOMIA }\end{array}$ & 0,6 & 0,5 & 0,5 & 0,5 & 0,8 & 0,4 & 0,8 & 2,7 & 14 \\
\hline UPG ZOOTECNIA & 0,4 & 0,3 & 0,4 & 0,3 & 0,3 & 0,3 & 0,6 & 2,6 & 15 \\
\hline
\end{tabular}

\section{Planeamiento estratégico a nivel de UEN}

\section{Misión de la UPGFIM}

La misión de la UPGFIM es formar especialistas e investigadores humanistas e innovadores de las tecnologías para la producción industrial y aplicación energética mediante la mejora de la productividad del capital natural y la protección del medio ambiente.

\section{Visión de la UPGFIM}

La Visión UPGFIM es ser una unidad académica innovadora y ecológica, generadora de tecnología limpia para liderar el desarrollo tecnológico regional.

\section{Estrategia de la UPGFIM}

La estrategia fundamental de la UPGFIM es la eliminación de todo tipo de desperdicios, por tanto, la formación de los investigadores estará basada en el desarrollo de la tesis durante sus estudios, logrando concluir el borrador de tesis en el cuarto semestre eliminando el desperdicio de tiempo y capacidades.

\section{Grados que otorga:}

- Magíster en Ingeniería Mecánica con mención en Diseño de Tecnologías Apropiadas

- Magíster en Ingeniería Mecánica con mención en Gestión del Mantenimiento y la Sostenibilidad

- Magíster en Tecnología Energética

Análisis Interno: Organigrama actual de la Unidad de Posgrado de la Facultad de Ingeniería Mecánica 


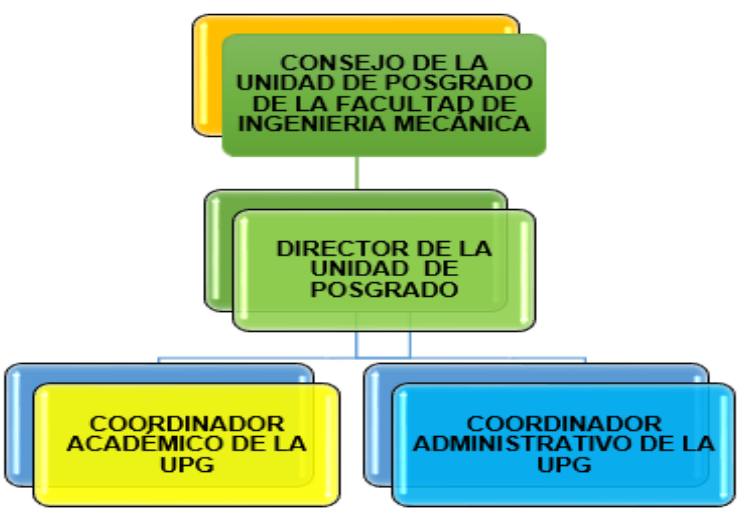

Diagrama de Contexto: Stakeholders Internos y Externos

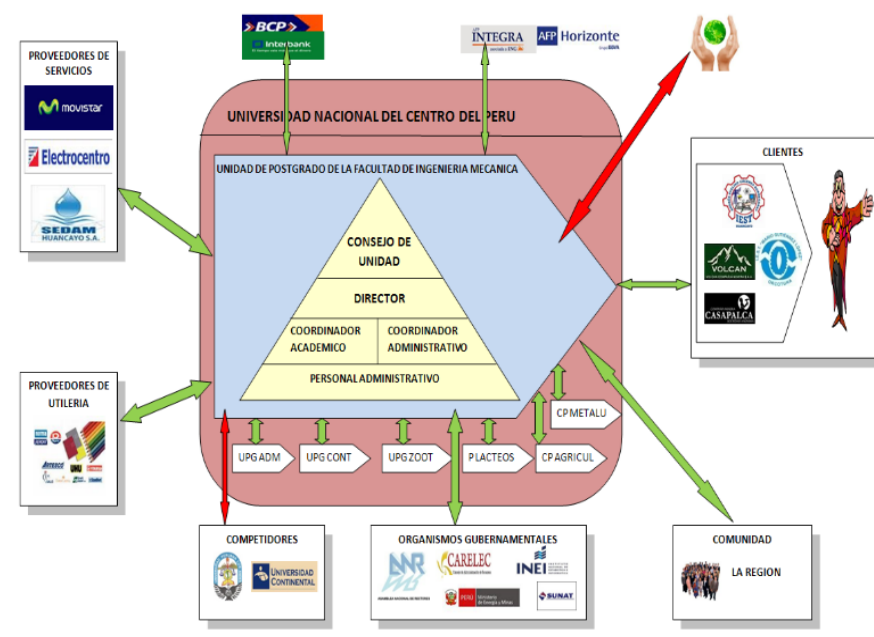

Cadena de Valor de la Unidad de Posgrado de la Facultad de Ingeniería Mecánica

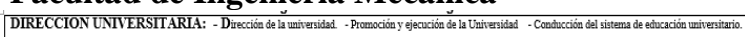

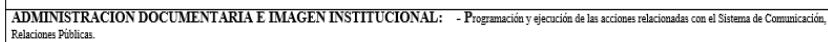

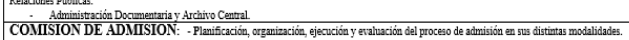

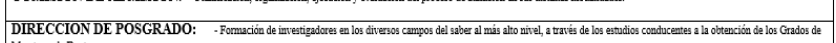

Maeasoy deb Doctar.

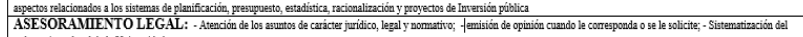

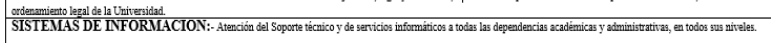

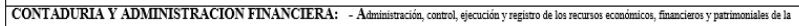

Uaberishat

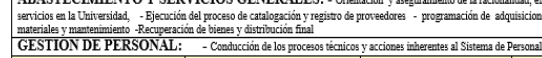

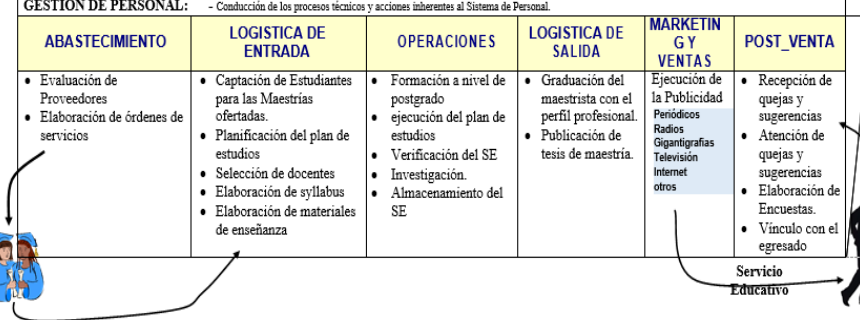

Tecnologías actuales

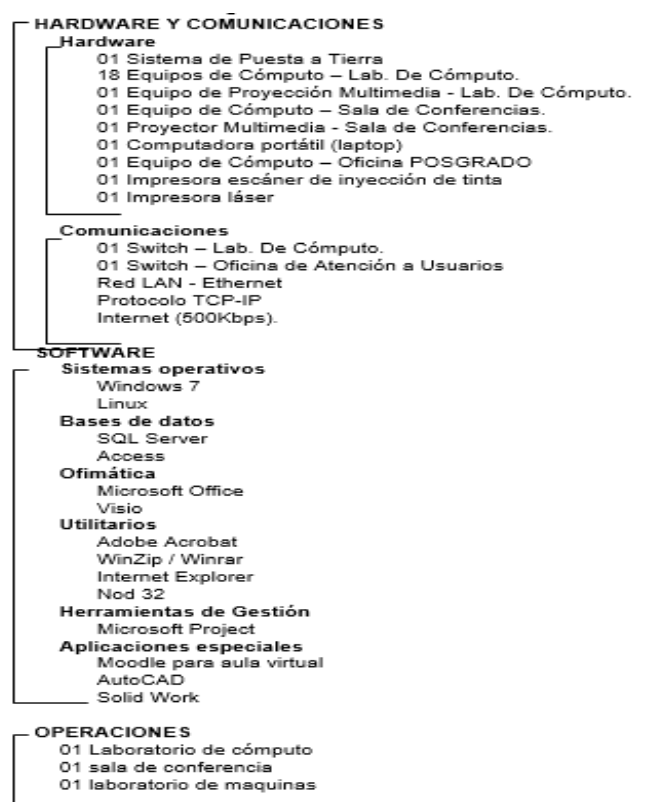

Matriz para perfil de áreas funcionales (PAF)

\begin{tabular}{|c|c|c|c|c|c|c|c|c|c|c|}
\hline \multirow[b]{2}{*}{ Área Funcional } & \multicolumn{3}{|c|}{$\begin{array}{l}\text { FORTAL } \\
\text { EZAS }\end{array}$} & \multicolumn{3}{|c|}{$\begin{array}{l}\text { DEBILI } \\
\text { DADES }\end{array}$} & \multicolumn{4}{|c|}{ IMPACTO } \\
\hline & 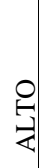 & $\stackrel{0}{\stackrel{0}{\mid}}$ & $\stackrel{0}{\stackrel{0}{\infty}}$ & \begin{tabular}{l}
0 \\
\multirow{2}{*}{}
\end{tabular} & $\begin{array}{l}\stackrel{0}{\rho} \\
\stackrel{9}{\Sigma}\end{array}$ & 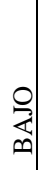 & 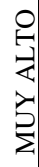 & \begin{tabular}{l}
0 \\
\multirow{2}{*}{} \\
\multirow{2}{*}{}
\end{tabular} & $\begin{array}{l}\stackrel{0}{a} \\
\text { 国 } \\
\Sigma\end{array}$ & 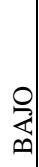 \\
\hline \multicolumn{11}{|l|}{ ABASTECIMIENTO } \\
\hline Evaluación de Proveedores & & $\mathrm{X}$ & & & & & $\mathrm{X}$ & & & \\
\hline $\begin{array}{l}\text { Elaboración de órdenes de } \\
\text { servicios }\end{array}$ & & & $\mathrm{X}$ & & & & & $\mathrm{X}$ & & \\
\hline Ejecución de la Publicidad & & & $X$ & & & & & $\mathrm{X}$ & & \\
\hline \multicolumn{11}{|l|}{ LOGISTICA DE ENTRADA } \\
\hline $\begin{array}{l}\text { Captación de Estudiantes para } \\
\text { las Maestrías ofertadas. }\end{array}$ & & $\mathrm{X}$ & & & & & & $\mathrm{X}$ & & \\
\hline $\begin{array}{l}\text { Planificación del plan de } \\
\text { estudios }\end{array}$ & & $\mathrm{X}$ & & & & & & $\mathrm{X}$ & & \\
\hline Selección de docentes & & $\mathrm{X}$ & & & & & & & $\mathrm{X}$ & \\
\hline Elaboración de syllabus & & & & & $\mathrm{X}$ & & & & $\mathrm{X}$ & \\
\hline $\begin{array}{l}\text { Elaboración de materiales de } \\
\text { enseñanza }\end{array}$ & & $\mathrm{X}$ & & & & & & & $\mathrm{X}$ & \\
\hline \multicolumn{11}{|l|}{ OPERACIONES } \\
\hline Formación a nivel de postgrado & $\mathrm{X}$ & & & & & & $\mathrm{X}$ & & & \\
\hline Ejecución del plan de estudios & & $\mathrm{X}$ & & & & & & $\mathrm{X}$ & & \\
\hline Verificación del SE & & $\mathrm{X}$ & & & & & & $\mathrm{X}$ & & \\
\hline Investigación & & & $\mathrm{X}$ & & & & & & $\mathrm{X}$ & \\
\hline Almacenamiento del SE & & & & $\mathrm{X}$ & & & $\mathrm{X}$ & & & \\
\hline \multicolumn{11}{|l|}{ LOGÍSTICA DE SALIDA } \\
\hline $\begin{array}{l}\text { Graduación del maestrista con } \\
\text { el perfil profesional. }\end{array}$ & & $\mathrm{X}$ & & & & & $\mathrm{X}$ & & & \\
\hline $\begin{array}{l}\text { Publicación de tesis de } \\
\text { maestría. }\end{array}$ & & $\mathrm{X}$ & & & & & $\mathrm{X}$ & & & \\
\hline \multicolumn{11}{|l|}{ POST VENTA } \\
\hline $\begin{array}{l}\text { Recepción de quejas y } \\
\text { sugerencias }\end{array}$ & & & $\mathrm{X}$ & & & & & $\mathrm{X}$ & & \\
\hline
\end{tabular}




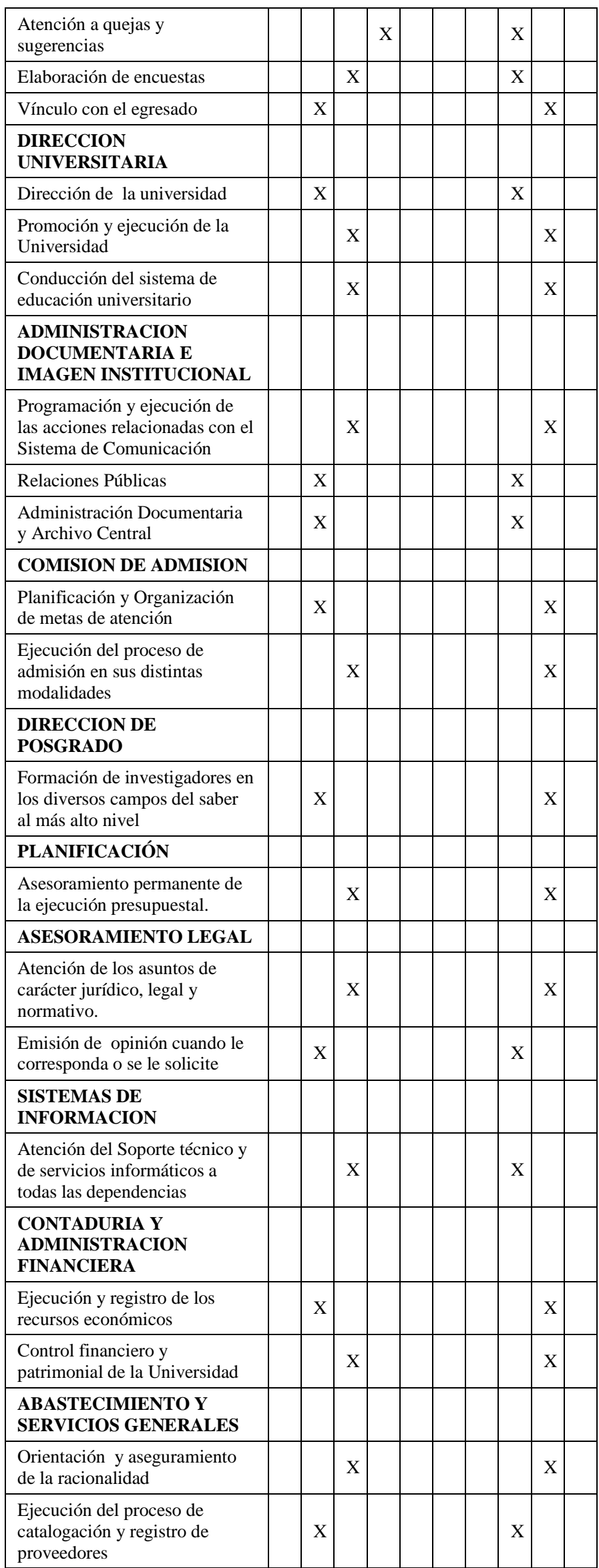

\begin{tabular}{|l|l|l|l|l|l|l|l|l|l|l|}
\hline $\begin{array}{l}\text { Programación de adquisiciones } \\
\text { y almacenamiento }\end{array}$ & & $\mathrm{X}$ & & & & & & $\mathrm{X}$ & & \\
\hline GESTION DE PERSONAL & & & & & & & & & & \\
\hline $\begin{array}{l}\text { Planificación del Cuadro de } \\
\text { Asignación del Personal }\end{array}$ & & $\mathrm{X}$ & & & & & & $\mathrm{X}$ & & \\
\hline $\begin{array}{l}\text { Control de asistencia del } \\
\text { personal }\end{array}$ & & $\mathrm{x}$ & & & & & & $\mathrm{X}$ & & \\
\hline
\end{tabular}

Análisis externo: POAM para fuerzas competitivas

\begin{tabular}{|c|c|c|c|c|c|c|c|c|c|c|}
\hline \multirow[b]{2}{*}{ Fuerzas Competitivas } & \multicolumn{3}{|c|}{$\begin{array}{c}\text { OPORT } \\
\text { UNIDAD } \\
\text { ES } \\
\end{array}$} & \multicolumn{3}{|c|}{$\begin{array}{l}\text { AMENA } \\
\text { ZAS }\end{array}$} & \multicolumn{4}{|c|}{ IMPACTO } \\
\hline & $\begin{array}{l}0 \\
\stackrel{9}{4} \\
\stackrel{4}{4}\end{array}$ & $\frac{0}{\mathrm{a}}$ & 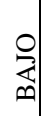 & 号 & $\begin{array}{l}0 \\
\stackrel{0}{2} \\
\dot{2}\end{array}$ & 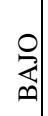 & 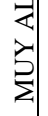 & 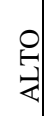 & $\frac{0}{\stackrel{0}{n}}$ & 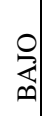 \\
\hline \multicolumn{11}{|l|}{ COMPETIDORES } \\
\hline Crecimiento del mercado & $\mathrm{X}$ & & & & & & & $\mathrm{X}$ & & \\
\hline $\begin{array}{l}\text { Costos fijos y/o barreras de } \\
\text { entradas }\end{array}$ & & $\mathrm{X}$ & & & & & & & $\mathrm{X}$ & \\
\hline $\begin{array}{l}\text { Disponibilidad de tecnologías y } \\
\text { sistemas de información }\end{array}$ & $\mathrm{X}$ & & & & & & $\mathrm{X}$ & & & \\
\hline Servicios diferenciados & & $\mathrm{X}$ & & & & & & $\mathrm{X}$ & & \\
\hline \multicolumn{11}{|l|}{ CLIENTES } \\
\hline Número de clientes & $\mathrm{X}$ & & & & & & $\mathrm{X}$ & & & \\
\hline $\begin{array}{l}\text { Bajos costos de comunicación } \\
\text { con proveedores }\end{array}$ & $\mathrm{X}$ & & & & & & $\mathrm{X}$ & & & \\
\hline Identificación con el servicio & & $\mathrm{X}$ & & & & & & $\mathrm{X}$ & & \\
\hline $\begin{array}{l}\text { Exigencia en la calidad del } \\
\text { servicio }\end{array}$ & & & & $\mathrm{X}$ & & & $\mathrm{X}$ & & & \\
\hline \multicolumn{11}{|l|}{ PROVEEDORES } \\
\hline Número de proveedores & & $\mathrm{X}$ & & & & & & $\mathrm{X}$ & & \\
\hline $\begin{array}{l}\text { Disponibilidad de proveedores } \\
\text { potenciales }\end{array}$ & & $\mathrm{X}$ & & & & & & $\mathrm{X}$ & & \\
\hline $\begin{array}{l}\text { Calidad del servicio que } \\
\text { brindan }\end{array}$ & & & $\mathrm{X}$ & & & & & & $\mathrm{X}$ & \\
\hline \multicolumn{11}{|l|}{$\begin{array}{l}\text { ORGANISMOS } \\
\text { GUBERNAMENTALES }\end{array}$} \\
\hline Tasa Impositivas & & & & $\mathrm{X}$ & & & & & $\mathrm{X}$ & \\
\hline Normatividad de la UNCP & & & & & & $\mathrm{X}$ & & & $\mathrm{X}$ & \\
\hline Situación actual del gobierno & & & & $\mathrm{X}$ & & & & $\mathrm{X}$ & & \\
\hline $\begin{array}{l}\text { Proyección de la imagen de la } \\
\text { institución en el Exterior }\end{array}$ & & $\mathrm{X}$ & & & & & & $\mathrm{X}$ & & \\
\hline Firma de Convenios & $\mathrm{X}$ & & & & & & $\mathrm{X}$ & & & \\
\hline Políticas económicas & & & & $\mathrm{X}$ & & & & & $\mathrm{X}$ & \\
\hline \multicolumn{11}{|l|}{$\begin{array}{l}\text { ENTIDADES } \\
\text { FINANCIERAS } \\
\end{array}$} \\
\hline Límite de crédito & & $\mathrm{X}$ & & & & & & $\mathrm{X}$ & & \\
\hline $\begin{array}{l}\text { Respaldo de Organismos } \\
\text { Internacionales }\end{array}$ & $\mathrm{X}$ & & & & & & $\mathrm{X}$ & & & \\
\hline \multicolumn{11}{|l|}{ MEDIO AMBIENTE } \\
\hline Variedad del clima & & & $\mathrm{X}$ & & & & & $\mathrm{X}$ & & \\
\hline Contaminación ambiental & & & & $\mathrm{X}$ & & & & $\mathrm{X}$ & & \\
\hline $\begin{array}{l}\text { Diversidad de Recursos } \\
\text { Naturales }\end{array}$ & $\mathrm{X}$ & & & & & & $\mathrm{X}$ & & & \\
\hline \multicolumn{11}{|l|}{$\begin{array}{l}\text { PROVEEDORES DE } \\
\text { SERVICIO }\end{array}$} \\
\hline Respaldo crediticio & & $\mathrm{X}$ & & & & & & $\mathrm{X}$ & & \\
\hline Crecimiento Económico & $\mathrm{X}$ & & & & & & $\mathrm{X}$ & & & \\
\hline \multicolumn{11}{|l|}{$\begin{array}{l}\text { ORGANISMOS } \\
\text { INTERNACIONALES }\end{array}$} \\
\hline Firma de Convenios & & $\mathrm{X}$ & & & & & & $\mathrm{X}$ & & \\
\hline Políticas económicas & & & $\mathrm{X}$ & & & & & & $\mathrm{X}$ & \\
\hline Nivel Académico & & $\mathrm{X}$ & & & & & & $\mathrm{X}$ & & \\
\hline \multicolumn{11}{|l|}{ SOCIOS ESTRATÉGICOS } \\
\hline Normatividad UNCP & & $\mathrm{X}$ & & & & & & $\mathrm{X}$ & & \\
\hline Desarrollo Tecnológico & & $\mathrm{X}$ & & & & & & $\mathrm{X}$ & & \\
\hline emanda $\mathrm{p}$ & & $\mathrm{X}$ & & & & & & & & \\
\hline
\end{tabular}




\begin{tabular}{|l|l|l|l|l|l|l|l|l|l|l|}
\hline Desarrollo Profesional & $\mathrm{X}$ & & & & & & $\mathrm{X}$ & & & \\
\hline
\end{tabular}

\section{Tendencia tecnológica}

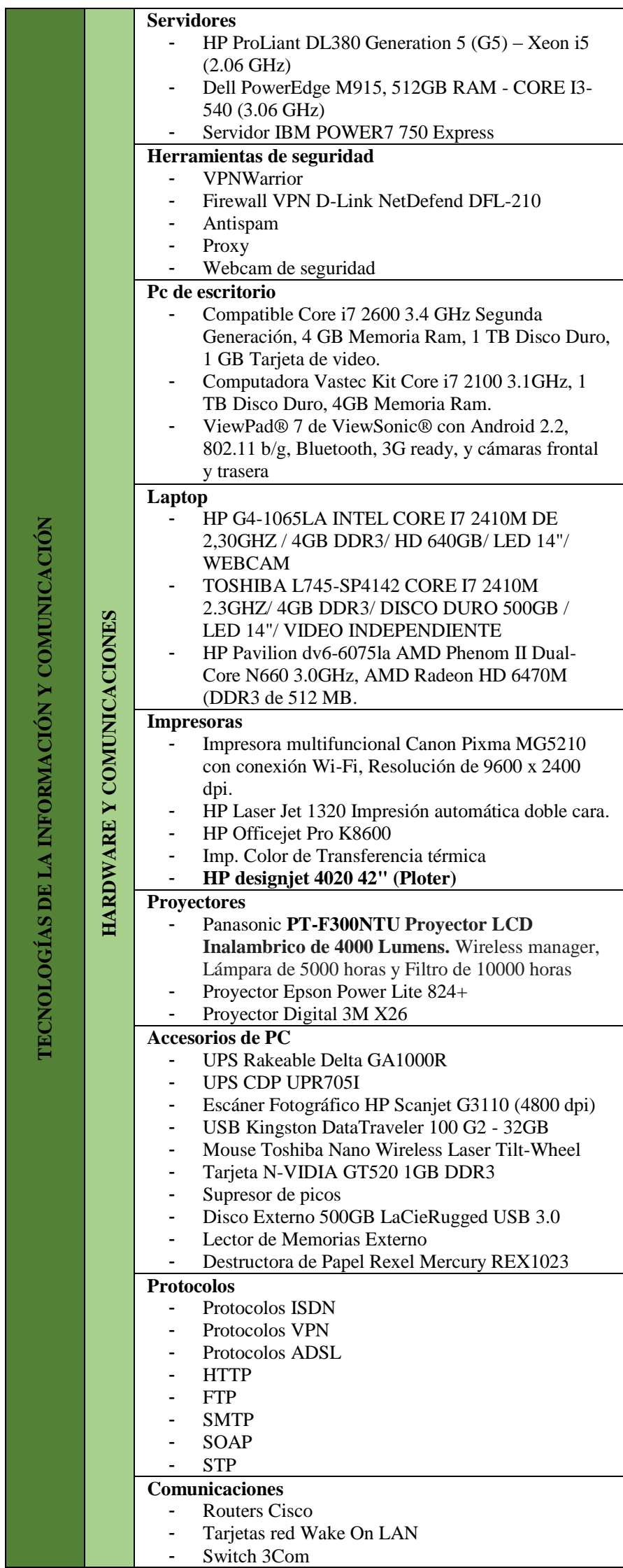

\begin{tabular}{|c|c|}
\hline & $\begin{array}{ll}- & \text { Switch D-Link } \\
\text { - } & \text { Access Point } \\
\text { - } & \text { Estaciones LAN } \\
\text { - } & \text { Central Telefónica } \\
\text { - } & \text { Teléfonos Corporativos (Nextel,Claro,Telefonica) } \\
\text { - } & \text { Telefonía VoIP } \\
\text { - } & \text { Tecnología Bluetooth } \\
\text { - } & \text { Tecnología Wifi -Wimax } \\
\end{array}$ \\
\hline & \begin{tabular}{cl}
\multicolumn{2}{l}{ SistemasOperativos } \\
- & MS Windows 7 \\
- & MS Windows 8 \\
- & Windows Server 2008 \\
- & UBUNTU v. 12.04 \\
- & Android \\
\end{tabular} \\
\hline & \begin{aligned} & \multicolumn{2}{l}{ Base de Datos } \\
& - $\quad$ Oracle $11 \mathrm{G} \\
&$ - $\quad$ MS Access 2010 \\
& - DB2 \\
& - MS SQL Server 2008 \\
& - Postgree \end{aligned} \\
\hline & \begin{tabular}{cc}
\multicolumn{2}{l}{ Ofimática } \\
$-\quad$ Open Office 3.3 \\
$-\quad$ MS Office 2012 \\
\end{tabular} \\
\hline & $\begin{array}{cl}\text { Desarrollo de Aplicaciones de Escritorio } \\
\text { - } & \text { Visual Studio } 2012 \\
- & \text { VB\# } \\
- & \text { Netbeans } 7.0\end{array}$ \\
\hline & $\begin{array}{cl}\text { Desarrollo de Aplicaciones Web } \\
\text { - } & \text { Asp. Net } \\
- & \text { JSP } \\
- & \text { CGI } \\
- & \text { XML } \\
- & \text { JSF } \\
- & \text { Struts } \\
\end{array}$ \\
\hline & $\begin{array}{cl}\text { Desarrollo de Aplicaciones Móviles } \\
\text { - } & \text { J2ME Java Micro Edition } \\
\text { - } & \text { Android } \\
\text { - } & \text { iTunes } \\
\text { - } & \text { Windows Mobile } \\
\text { - } & \text { Symbian } \\
\end{array}$ \\
\hline & \begin{aligned} & \multicolumn{1}{l}{ Diseñadores } \\
& - Corel Draw X5 \\
& - Adobe Photoshop CS 5.5 \\
& - Adobe Ilustrator CS 5.5 \\
& - Adobe Fireworks CS 5.5 \\
& - Adobe Flash CS 5.5 \\
& - Adobe Dreamweaver CS 5.5\end{aligned} \\
\hline & $\begin{array}{cl}\text { Utilitarios } \\
\text { - } & \text { Internet Explorer } \\
\text { - } & \text { Google Chrome } \\
\text { - } & \text { Google Apps } \\
\text { - } & \text { Antivirus Panda } \\
\text { - } & \text { Antivirus Symantec } \\
\text { - } & \text { Antivirus Avast } \\
\text { - } & \text { Adobe Reader } 10.0 \\
\text { - } & \text { Nero } \\
\text { - } & \text { Skype } \\
\end{array}$ \\
\hline$\frac{\sqrt[c]{2}}{\frac{1}{2}}$ & $\begin{array}{cl}\text { Aplicaciones Informáticas } \\
\text { - } & \text { Blended Learning } \\
\text { - } & \text { Data mining } \\
\text { - } & \text { Agentes Inteligentes } \\
\text { - } & \text { Business Intelligence } \\
\text { - } & \text { Videoconferencia } \\
\text { - } & \text { Acceso Remoto } \\
\text { - } & \text { CRM } \\
\text { - } & \text { Sistemas Expertos } \\
\text { - } & \text { GPS } \\
\text { - } & \text { BSC } \\
\text { - } & \text { Teletrabajo } \\
\text { - } & \text { Cloud computing } \\
\end{array}$ \\
\hline
\end{tabular}




\begin{tabular}{|c|c|}
\hline & 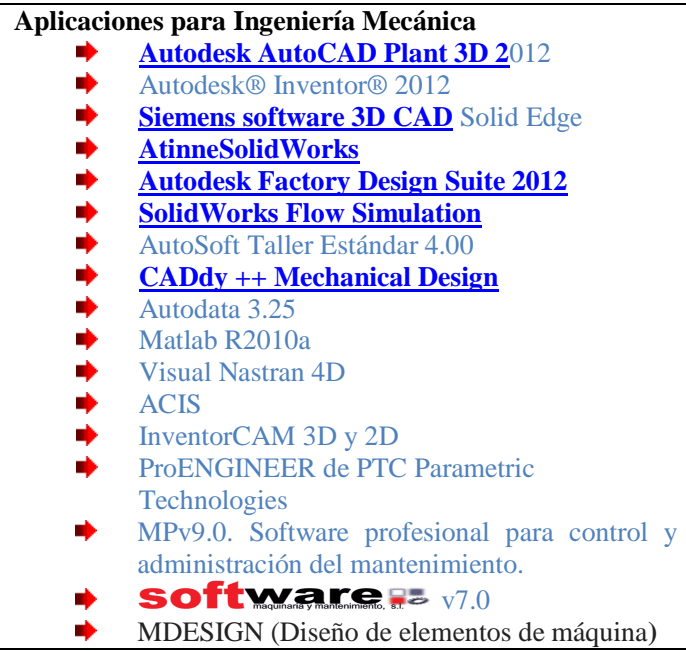 \\
\hline 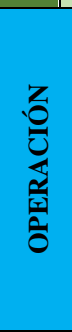 & $\begin{array}{ll}\text { - } & \text { Videoscopio VS36 } \\
\text { - } & \text { Equipo de Limpieza de Inyectores Diesel } \\
\text { - } & \text { Adaptadores de Limpieza } \\
\text { - } & \text { Equipo Electrónico } \\
\text { - } & \text { Laboratorio para inyectores } \\
\text { - } & \text { Lámpara de tiempo } \\
\text { - } & \text { Probador de sensores } \\
\text { - } & \text { Pulsador de Inyectores } \\
\text { - } & \text { Máquinas de Control Numérico } \\
\text { - } & \text { Maquinas de Oleohidraulica y Neumática } \\
\end{array}$ \\
\hline 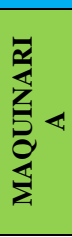 & $\begin{array}{ll}- & \text { Opel eléctrico 2013 } \\
- & \text { Smart ForTwo 2012 } \\
- & \text { Nissan JukeKuro 2012 } \\
- & \text { Motor del Renault Laguna } 2012 \\
- & \text { Mazda CX 5 } \\
- & \text { Renault Koleos 2012 } \\
- & \text { Motores eléctricos } \\
\end{array}$ \\
\hline
\end{tabular}

\section{Análisis DOFA}

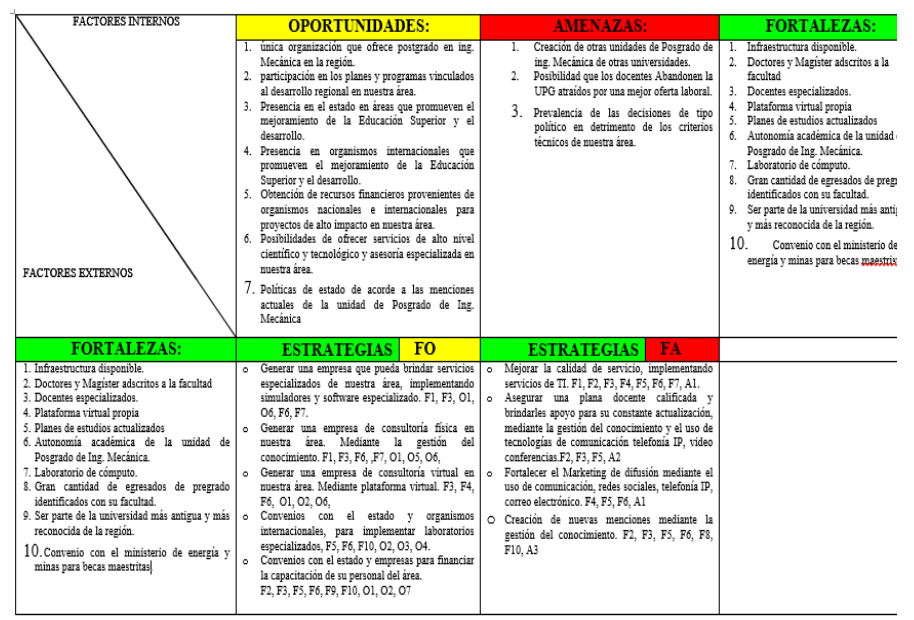

\begin{tabular}{|c|c|c|c|}
\hline 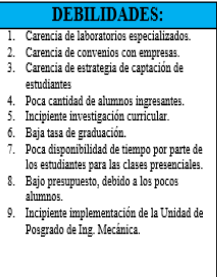 & 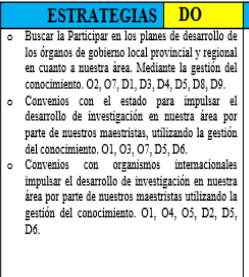 & 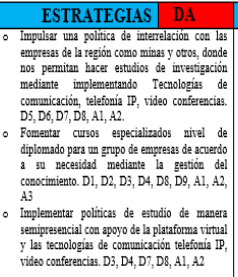 & 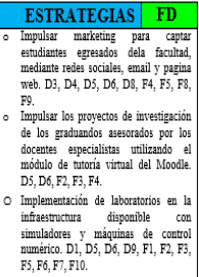 \\
\hline 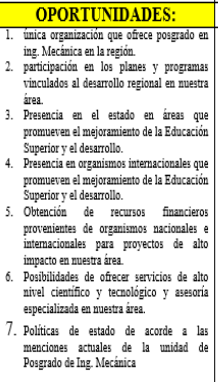 & & 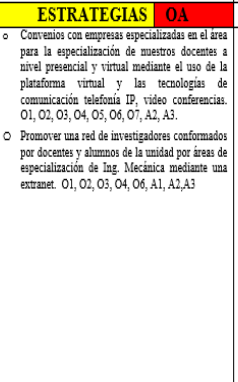 & \\
\hline
\end{tabular}

3. Direccionamiento Estratégico de la Unidad de Posgrado de la Facultad de Ingeniería Mecánica de la UNCP

Misión de la UPGFIM.- La misión de la UPGFIM es formar especialistas e investigadores humanistas e innovadores de las tecnologías para la producción industrial y aplicación energética mediante la mejora de la productividad del capital natural y la protección del medio ambiente.

Visión de la UPGFIM.- La Visión UPGFIM es ser una unidad académica innovadora y ecológica, generadora de tecnología limpia para liderar el desarrollo tecnológico regional.

Estrategia de la UPGFIM.- La estrategia fundamental de la UPGFIM es la eliminación de todo tipo de desperdicios, por tanto, la formación de los investigadores estará basada en el desarrollo de la tesis durante sus estudios, logrando concluir el borrador de tesis en el cuarto semestre eliminando el desperdicio de tiempo y capacidades.

\section{Objetivos estratégicos para la UEN}

- Establecer un modelo de gestión integrado, interrelacionado la UPG de Ing. Mecánica con otras unidades estratégicas, tales como: la escuela de Posgrado y la oficina general de registros académicos.

- Implementar tecnología de intranet y extranet para potenciar la actividad académica y administrativa de la UPG de Ing. Mecánica.

- Incrementar la cantidad de estudiantes mediante marketing utilizando las TICs disponibles

- Promocionar nuevas maestrías de acuerdo a las necesidades empresariales considerando las últimas tecnologías de información.

Cadena de Valor Propuesto de la Unidad de Posgrado de la Facultad de Ingeniería Mecánica 


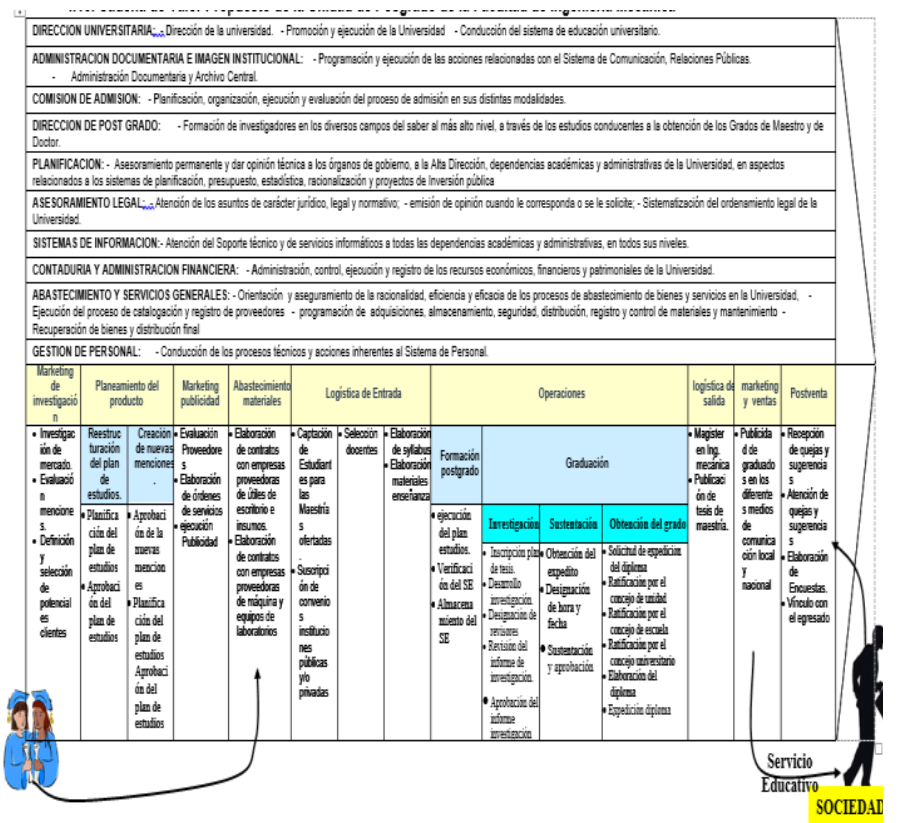

Organigrama propuesto de la Unidad de Posgrado de la Facultad de Ingeniería Mecánica

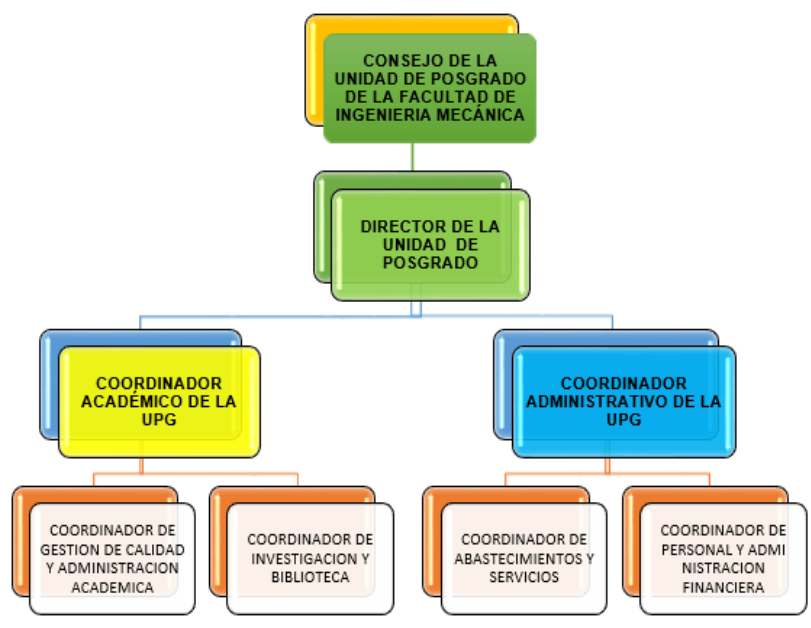

Estrategias utilizadas: básica(s) y de apoyo.

- Estrategias básicas

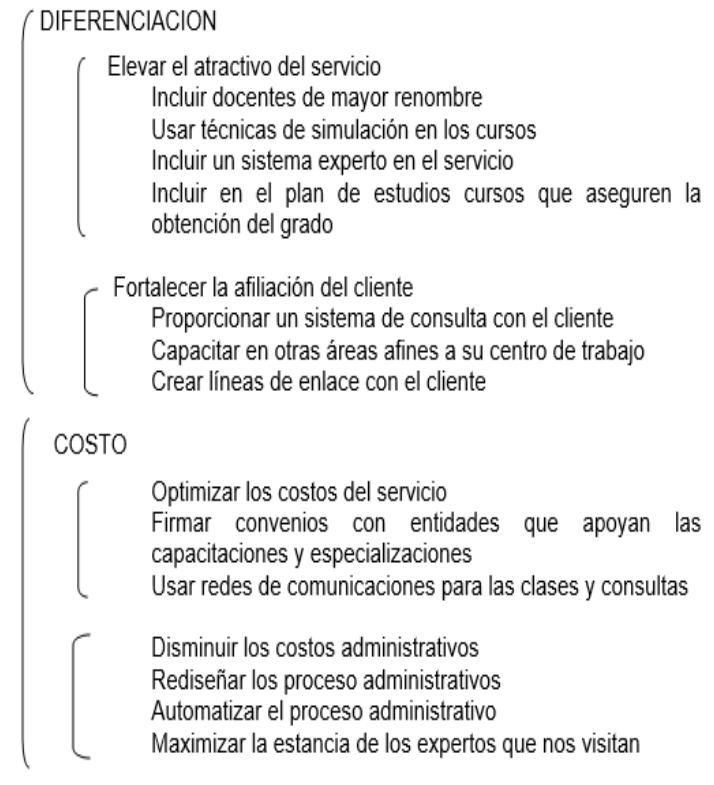

- $\quad$ Estrategias de apoyo

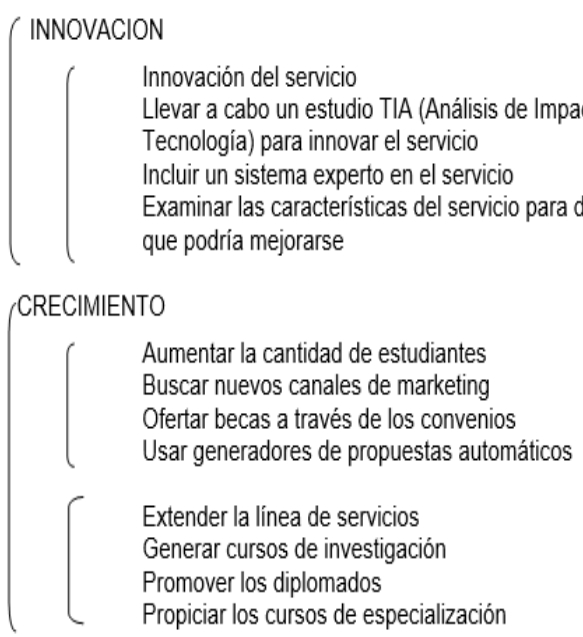

4. Plan de TI

Matriz Tecnologías de Información vs Componentes DOFA

\begin{tabular}{|c|c|c|c|}
\hline $\begin{array}{r}\text { COMPONE } \\
\text { NTES DOFA }\end{array}$ & $\begin{array}{c}\text { OBJETIVOS } \\
\text { DEL } \\
\text { NEGOCIO }\end{array}$ & 密 & อ \\
\hline
\end{tabular}




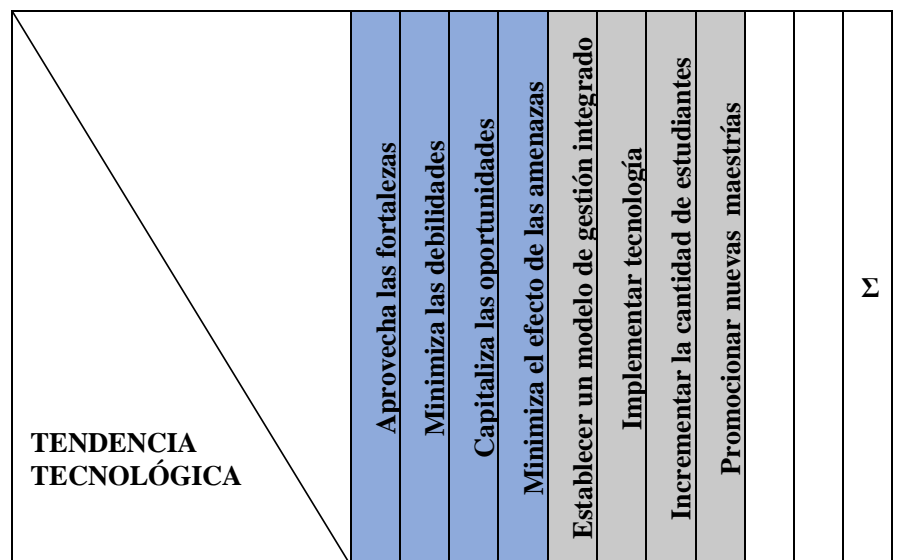

HARDWARE Y COMUNICACIONES

\begin{tabular}{|c|c|c|c|c|c|c|c|c|c|c|c|}
\hline Servidores & & & & & & & & & & & \\
\hline $\begin{array}{l}\text { HP ProLiant DL380 } \\
\text { Generation 5 (G5) - } \\
\text { Xeon i5 }(2.06 \mathrm{GHz}\end{array}$ & 3 & 3 & 1 & 3 & 1 & 3 & 3 & 3 & 3 & 5 & 28 \\
\hline $\begin{array}{lr}\text { Dell } & \text { PowerEdge } \\
\text { M915, 512GB RAM - } \\
\text { CORE } & \text { I3-540 } \\
\text { GHz }) & \end{array}$ & 3 & 3 & 3 & 3 & 3 & 3 & 3 & 3 & 3 & 5 & 32 \\
\hline $\begin{array}{lr}\text { Servidor } & \text { IBM } \\
\text { POWER7 } 750 \text { Express } \\
\end{array}$ & 3 & 3 & 3 & 5 & 5 & 5 & 3 & 3 & 3 & 5 & 38 \\
\hline $\begin{array}{l}\text { Herramientas de } \\
\text { seguridad }\end{array}$ & & & & & & & & & & & \\
\hline VPNWarriror & 3 & 3 & 3 & 3 & 3 & 3 & 3 & 3 & 3 & 3 & 30 \\
\hline $\begin{array}{l}\text { Firewall VPN D-Link } \\
\text { NetDefend DFL-210 }\end{array}$ & 5 & 5 & 3 & 5 & 5 & 3 & 5 & 3 & 3 & 3 & 35 \\
\hline Antispam & 3 & 3 & 3 & 3 & 3 & 3 & 3 & 3 & 3 & 3 & 30 \\
\hline Proxy & 3 & 3 & 3 & 3 & 3 & 3 & 3 & 3 & 1 & 3 & 28 \\
\hline Webcam de seguridad & 3 & 3 & 3 & 5 & 3 & 3 & 3 & 3 & 3 & 3 & 32 \\
\hline Pc de escritorio & & & & & & & & & & & \\
\hline $\begin{array}{l}\text { Compatible Core i7 } \\
2600 \text { 3.4 GHz Segunda } \\
\text { Generación, } 4 \text { GB } \\
\text { Memoria Ram, 1 TB } \\
\begin{array}{ll}\text { Disco Duro, 1 GB } \\
\text { Tarjeta de video }\end{array} \\
\end{array}$ & 3 & 3 & 1 & 3 & 3 & 3 & 3 & 3 & 5 & 3 & 30 \\
\hline $\begin{array}{l}\text { ComputadoraVastec } \\
\text { Kit Core i3 2100 } \\
3.1 \mathrm{GHz}, 500 \mathrm{~GB} \text { Disco } \\
\text { Duro, 4GB Memoria } \\
\text { Ram }\end{array}$ & 3 & 3 & 1 & 1 & 3 & 3 & 3 & 3 & 3 & 3 & 28 \\
\hline 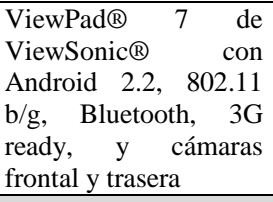 & 3 & 3 & 1 & 1 & 1 & 1 & 3 & 1 & 3 & 3 & 20 \\
\hline Laptop & & & & & & & & & & & \\
\hline $\begin{array}{l}\text { HP G4-1065LA } \\
\text { INTEL CORE I5 } \\
\text { 2410M DE 2,30GHZ / } \\
\text { 4GB DDR3/ HD } \\
\text { 640GB/ LED 14"/ } \\
\text { WEBCAM }\end{array}$ & 3 & 3 & 3 & 3 & 3 & 5 & 3 & 3 & 1 & 3 & 30 \\
\hline $\begin{array}{l}\text { TOSHIBA L745- } \\
\text { SP4142 CORE I5 } \\
2410 \mathrm{M} \text { 2.3GHZ/ 4GB } \\
\text { DDR3/ DISCO DURO } \\
\text { 500GB / LED 14"/ } \\
\text { VIDEO } \\
\text { INDEPENDIENTE }\end{array}$ & 3 & 3 & 3 & 3 & 1 & 3 & 3 & 3 & 1 & 3 & 26 \\
\hline $\begin{array}{l}\text { HP Pavilion dv6- } \\
\text { 6075la AMD Phenom } \\
\text { II Dual-Core N660 } \\
\text { 3.0GHz, AMD Radeon }\end{array}$ & 3 & 3 & 1 & 3 & 1 & 3 & 3 & 3 & 1 & 1 & 22 \\
\hline
\end{tabular}

\begin{tabular}{|c|c|c|c|c|c|c|c|c|c|c|c|}
\hline $\begin{array}{l}\text { HD 6470M (DDR3 de } \\
512 \mathrm{MB}\end{array}$ & & & & & & & & & & & \\
\hline Impresoras & & & & & & & & & & & \\
\hline $\begin{array}{l}\text { Impresora } \\
\text { multifuncional Canon } \\
\text { Pixma MG5210 con } \\
\text { conexión Wi-Fi, } \\
\text { Resolución de } 9600 \text { x } \\
2400 \text { dpi }\end{array}$ & 3 & 3 & 1 & 1 & 3 & 1 & 3 & 1 & 3 & 5 & 24 \\
\hline $\begin{array}{l}\text { HP Laser Jet } 1320 \\
\text { Impresión automática } \\
\text { doble cara }\end{array}$ & 3 & 3 & 1 & 1 & 5 & 3 & 3 & 3 & 5 & 5 & 32 \\
\hline $\begin{array}{l}\text { HP Officejet Pro } \\
\text { K8600 }\end{array}$ & 3 & 3 & 1 & 1 & 3 & 1 & 3 & 1 & 1 & 5 & 22 \\
\hline $\begin{array}{l}\text { Imp. Color de } \\
\text { Transferencia térmica }\end{array}$ & 3 & 3 & 1 & 1 & 3 & 1 & 3 & 1 & 3 & 5 & 24 \\
\hline $\begin{array}{l}\text { HP designjet } 402042 " \\
\text { (Ploter) }\end{array}$ & 5 & 5 & 5 & 5 & 5 & 5 & 5 & 5 & 5 & 5 & 50 \\
\hline
\end{tabular}

\begin{tabular}{|c|} 
Proyectores \\
\hline Panasonic
\end{tabular}

Panasonic PT-

F300NTU

Proyector LCD

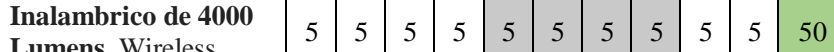

Lumens. Wireless

manager, Lámpara de

5000 horas y Filtro de

10000 horas

\begin{tabular}{|l|l|l|l|l|l|l|l|l|l|l|l|}
\hline $\begin{array}{l}\text { Proyector Epson } \\
\text { Power Lite 824+ }\end{array}$ & 3 & 3 & 1 & 3 & 5 & 3 & 3 & 3 & 5 & 5 & 35 \\
\hline $\begin{array}{l}\text { Proyector Digital 3M } \\
\text { X26 }\end{array}$ & 3 & 3 & 1 & 1 & 3 & 1 & 3 & 3 & 3 & 5 & 27 \\
\hline
\end{tabular}

X26

\begin{tabular}{|l|c|c|c|c|c|c|c|c|c|c|c|}
\hline Accesorios de PC & & & & & & & & & & & \\
\hline UPS Rakeable Delta & 3 & 3 & 1 & 1 & 3 & 3 & 3 & 3 & 3 & 3 & 26 \\
\hline
\end{tabular}

GA1000R

\begin{tabular}{|l|l|l|l|l|l|l|l|l|l|l|l|}
\hline UPS CDP UPR705I & 5 & 5 & 3 & 3 & 5 & 5 & 5 & 3 & 3 & 3 & 39 \\
\hline
\end{tabular}

\begin{tabular}{|l|l|l|l|l|l|l|l|l|l|l|l|}
\hline Escáner Fotográfico & 3 & 3 & 1 & 1 & 3 & 3 & 3 & 3 & 3 & 3 & 26
\end{tabular}

HP Scanjet G3110

(4800 dpi)

USB Kingston

DataTraveler $100 \mathrm{G} 2$ -

32GB

\begin{tabular}{|l|l|l|l|l|l|l|l|l|l|l|l|}
\hline Mouse Toshiba Nano & 3 & 3 & 3 & 3 & 3 & 5 & 5 & 5 & 3 & 3 & 36
\end{tabular}

Wireless Laser Tilt-

Wheel

Tarjeta N-VIDIA

GT520 1GB DDR3

\begin{tabular}{|l|l|l|l|l|l|l|l|l|l|l|l|}
\hline Supresor de picos & 3 & 3 & 1 & 1 & 1 & 3 & 3 & 3 & 3 & 3 & 24 \\
\hline
\end{tabular}

\begin{tabular}{l|l|l|l|l|l|l|lllllll}
\hline Disco Externo 500GB & 5 & 3 & 3 & 3 & 1 & 3 & 3 & 3 & 3 & 3 & 30
\end{tabular}

LaCieRugged USB 3.0

\begin{tabular}{|l|l|l|l|l|l|l|l|l|l|l|l|}
\hline Lector de Memorias & 5 & 3 & 3 & 3 & 3 & 3 & 3 & 3 & 3 & 5 & 34
\end{tabular}

Externo

\begin{tabular}{|l|l|l|l|l|l|l|l|l|l|l|l|}
\hline Destructora de Papel & 3 & 3 & 1 & 3 & 3 & 3 & 3 & 3 & 3 & 3 & 28 \\
\hline
\end{tabular}

Rexel Mercury

REX1023

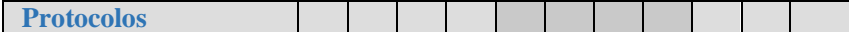

\begin{tabular}{|l|l|l|l|l|l|l|l|l|l|l|l|}
\hline Protocolos ISDN & 3 & 3 & 3 & 3 & 5 & 3 & 3 & 3 & 3 & 5 & 34 \\
\hline Proc
\end{tabular}

\begin{tabular}{|l|l|l|l|l|l|l|l|l|l|l|l|}
\hline Protocolos VPN & 3 & 3 & 1 & 1 & 5 & 3 & 3 & 3 & 3 & 5 & 32 \\
\hline
\end{tabular}

\begin{tabular}{|l|l|l|l|l|l|llllll|l|l|}
\hline Protocolos ADSL & 3 & 3 & 3 & 3 & 1 & 3 & 3 & 3 & 3 & 5 & 30 \\
\hline
\end{tabular}

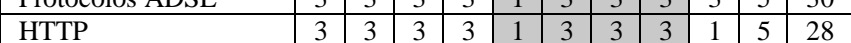

\begin{tabular}{|l|l|l|l|l|l|l|l|l|l|l|l|}
\hline FTP & 3 & 3 & 3 & 3 & 1 & 3 & 3 & 3 & 1 & 5 & 28 \\
\hline SMTP & 3 & 3 & 3 & 3 & 3 & 3 & 3 & 3 & 3 & 5 & 32 \\
\hline
\end{tabular}

\begin{tabular}{|c|c|c|c|c|c|c|c|c|c|c|c|}
\hline SMTP & 3 & 3 & 3 & 3 & 3 & 3 & 3 & 3 & 3 & 5 & 32 \\
\hline SOA & 3 & 3 & 3 & 3 & 3 & 1 & 3 & 3 & 3 & 5 & \\
\hline
\end{tabular}

STP

Comunicaciones

Routers Cisco

\begin{tabular}{llllllllllllll}
3 & 3 & 3 & 3 & 5 & 3 & 3 & 3 & 3 & 3 & 32 \\
\hline
\end{tabular}

LAN

\begin{tabular}{|l|l|l|l|l|l|l|l|l|l|l|l|}
\hline Switch 3Com & 3 & 3 & 1 & 3 & 3 & 3 & 3 & 3 & 3 & 3 & 28 \\
\hline Swich D-Link & 3 & 3 & 1 & 1 & 3 & 3 & 3 & 3 & 3 & 3 & 26 \\
\hline
\end{tabular}

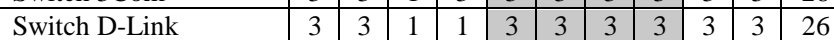

\begin{tabular}{|l|l|l|l|l|l|l|l|l|l|l|l|l}
\hline Access Point & 3 & 3 & 3 & 3 & 3 & 5 & 5 & 5 & 3 & 3 & 36 \\
\hline
\end{tabular}

\begin{tabular}{|l|l|l|l|l|l|lllllll}
\hline Estaciones LAN & 3 & 3 & 3 & 1 & 1 & 1 & 3 & 3 & 3 & 3 & 24 \\
\hline
\end{tabular}

Central Telefónica 


\begin{tabular}{|c|c|c|c|c|c|c|c|c|c|c|c|}
\hline $\begin{array}{l}\text { Teléfonos } \\
\text { Corporativos } \\
\text { (Nextel,Claro, } \\
\text { Movistar) }\end{array}$ & 3 & 3 & 1 & 1 & 1 & 1 & 3 & 3 & 3 & 3 & 22 \\
\hline Telefonía VoIP & 3 & 3 & 1 & 1 & 1 & 1 & 1 & 3 & 3 & 3 & 20 \\
\hline Tecnología Bluetooth & 3 & \begin{tabular}{|l|l|}
1 \\
\end{tabular} & 1 & \begin{tabular}{|l|l|l|}
1 \\
\end{tabular} & 1 & 1 & 1 & \begin{tabular}{|l|l|}
1 \\
\end{tabular} & 3 & 3 & 16 \\
\hline $\begin{array}{l}\text { Tecnología Wifi - } \\
\text { Wimax }\end{array}$ & 3 & 3 & 1 & 1 & 1 & 3 & 3 & 3 & 3 & 3 & 24 \\
\hline \multicolumn{12}{|l|}{ SOFTWARE } \\
\hline \multicolumn{12}{|l|}{ Sistemas Operativos } \\
\hline $\begin{array}{l}\text { MS Windows XP } \\
\text { Professional SP3 }\end{array}$ & 3 & 3 & 3 & 3 & 3 & 3 & 3 & 3 & 3 & 3 & 30 \\
\hline MS Windows 8 & 3 & 5 & 5 & 5 & 5 & 5 & 5 & 5 & 5 & 3 & 46 \\
\hline MS Windows 7 & 3 & 5 & 5 & 5 & 5 & 5 & 5 & 5 & 5 & 3 & 46 \\
\hline Windows Server 2008 & 3 & 3 & 3 & 3 & 5 & 5 & 5 & 5 & 5 & 3 & 40 \\
\hline Linux Red Hat & 3 & 3 & 3 & 3 & 3 & 3 & 3 & 3 & 3 & 3 & 30 \\
\hline Linux Ubuntu & 3 & 1 & 1 & 3 & 3 & 3 & 3 & 3 & 3 & 3 & 26 \\
\hline Linux Suse & 3 & 1 & 1 & 3 & 3 & 3 & 3 & 3 & 3 & 3 & 26 \\
\hline Android & 3 & 1 & 1 & 3 & 3 & 3 & 3 & 3 & 3 & 3 & 26 \\
\hline \multicolumn{12}{|l|}{ Base de Datos } \\
\hline Oracle 11G & 3 & 3 & 3 & 3 & 3 & 3 & 5 & 3 & 3 & 3 & 32 \\
\hline MS Access 2010 & 3 & 3 & 3 & 3 & 3 & 3 & 3 & 3 & 3 & 1 & 28 \\
\hline DB2 & 3 & 1 & 1 & 3 & 3 & 3 & 3 & 3 & 3 & 3 & 26 \\
\hline MS SQL Server 2008 & 3 & 3 & 3 & 5 & 5 & 5 & 5 & 5 & 5 & 3 & 42 \\
\hline Postgree & 3 & 1 & 1 & 1 & 1 & 3 & 3 & 3 & 3 & 3 & 22 \\
\hline MySQL & 3 & 3 & 1 & 1 & 3 & 3 & 3 & 3 & 1 & 3 & 24 \\
\hline \multicolumn{12}{|l|}{ Ofimática } \\
\hline Microsoft Office 2010 & 3 & 5 & 5 & 5 & 5 & 5 & 5 & 5 & 5 & 3 & 46 \\
\hline Open Office & 3 & 3 & 3 & 5 & 5 & 5 & 5 & 3 & 3 & 3 & 38 \\
\hline \multicolumn{12}{|l|}{$\begin{array}{l}\text { Desarrollo de } \\
\text { aplicaciones de } \\
\text { escritorio }\end{array}$} \\
\hline Visual Studio 2012 & 3 & 5 & 3 & 5 & 5 & 5 & 3 & 3 & 5 & 3 & 40 \\
\hline VB\# & 3 & 3 & 3 & 5 & 3 & 5 & 3 & 3 & 5 & 3 & 36 \\
\hline Netbeans 7.0 & 3 & 5 & 3 & 5 & 5 & 5 & 3 & 3 & 5 & 3 & 40 \\
\hline \multicolumn{12}{|l|}{$\begin{array}{l}\text { Desarrollo de } \\
\text { aplicaciones de Web }\end{array}$} \\
\hline Asp. Net & 3 & 5 & 3 & 5 & 5 & 5 & 3 & 3 & 5 & 3 & 40 \\
\hline JSP & 3 & 3 & 3 & 5 & 5 & 3 & 3 & 3 & 5 & 3 & 36 \\
\hline CGI & 3 & \begin{tabular}{|c|}
3 \\
\end{tabular} & 3 & 5 & 3 & 3 & 3 & 3 & 5 & 3 & 34 \\
\hline XML & 3 & 5 & 3 & 5 & 5 & 5 & 3 & 3 & 5 & 3 & 40 \\
\hline JSF & 3 & 5 & 3 & 5 & 5 & 5 & 3 & 3 & 5 & 3 & 40 \\
\hline Struts & 3 & 5 & 3 & 5 & 5 & 5 & 3 & 5 & 5 & 3 & 42 \\
\hline \multicolumn{12}{|l|}{$\begin{array}{l}\text { Desarrollo de } \\
\text { aplicaciones de } \\
\text { Móviles }\end{array}$} \\
\hline $\begin{array}{l}\text { J2ME Java Micro } \\
\text { Edition }\end{array}$ & 3 & 5 & 3 & 5 & 5 & 5 & 3 & 5 & 5 & 3 & 42 \\
\hline Android & 3 & 5 & 3 & 5 & 3 & 5 & 3 & 5 & 5 & 3 & 40 \\
\hline iTunes & 3 & 3 & 3 & 5 & 3 & 5 & 3 & 5 & 5 & 3 & 38 \\
\hline Windows Mobile & 3 & 5 & 3 & 5 & 5 & 5 & 3 & 5 & 5 & 3 & 42 \\
\hline Symbian & 3 & 5 & 3 & 5 & 5 & 5 & 3 & 3 & 5 & 3 & 40 \\
\hline \multicolumn{12}{|l|}{ Diseñadores } \\
\hline Corel Draw X5 & 3 & 3 & 3 & 1 & 3 & 3 & 1 & 1 & 3 & 3 & 24 \\
\hline $\begin{array}{l}\text { Adobe Photoshop CS } \\
5.5\end{array}$ & 1 & 1 & 1 & 1 & 3 & 3 & 1 & 1 & 1 & 3 & 16 \\
\hline $\begin{array}{l}\text { Adobe Ilustrator CS } \\
5.5\end{array}$ & 3 & 3 & 3 & 3 & 3 & 5 & 1 & 1 & 3 & 5 & 30 \\
\hline $\begin{array}{l}\text { Adobe Fireworks CS } \\
5.5\end{array}$ & 1 & 1 & 1 & 1 & 3 & 3 & 1 & 1 & 1 & 3 & 16 \\
\hline Adobe Flash CS 5.5 & 1 & \begin{tabular}{|l|}
1 \\
\end{tabular} & 1 & 1 & 3 & 3 & 3 & \begin{tabular}{|l|}
1 \\
\end{tabular} & 1 & 3 & 18 \\
\hline $\begin{array}{l}\text { Adobe Dreamweaver } \\
\text { CS } 5.5\end{array}$ & 1 & 1 & 1 & 1 & 3 & 3 & 3 & 1 & 1 & 3 & 18 \\
\hline \multicolumn{12}{|l|}{ Utilitarios } \\
\hline Internet Explorer & 3 & 3 & 5 & 5 & 5 & 5 & 5 & 5 & 5 & 3 & 44 \\
\hline GoogleChrome & 3 & 3 & 3 & 3 & 3 & 5 & 5 & 5 & 3 & 3 & 36 \\
\hline Google Apps & 3 & 3 & 3 & 3 & 3 & 3 & 3 & 3 & 3 & 3 & 30 \\
\hline Mozilla & 3 & 3 & 3 & 1 & 3 & 3 & 3 & 3 & 3 & 3 & 28 \\
\hline
\end{tabular}

\begin{tabular}{|l|c|c|c|c|c|c|c|c|c|c|c|}
\hline Antivirus Panda & 3 & 3 & 1 & 1 & 1 & 1 & 3 & 3 & 3 & 3 & 22 \\
\hline Antivirus Symantec & 3 & 3 & 3 & 5 & 3 & 5 & 3 & 3 & 3 & 3 & 31 \\
\hline Antivirus Avast & 3 & 1 & 1 & 1 & 3 & 3 & 3 & 3 & 3 & 3 & 24 \\
\hline Adobe Reader 10.0 & 3 & 1 & 1 & 1 & 1 & 1 & 1 & 3 & 3 & 3 & 18 \\
\hline Nero & 3 & 3 & 5 & 5 & 5 & 5 & 5 & 5 & 5 & 3 & 44 \\
\hline Skype & 3 & 3 & 3 & 3 & 3 & 5 & 5 & 5 & 3 & 3 & 36 \\
\hline $\begin{array}{l}\text { Aplicaciones } \\
\text { informáticas }\end{array}$ & & & & & & & & & & & \\
\hline $\begin{array}{l}\text { Blended Learning } \\
\text { Bence }\end{array}$ & 3 & 3 & 5 & 5 & 3 & 3 & 3 & 3 & 3 & 5 & 36 \\
\hline
\end{tabular}

\begin{tabular}{|l|l|l|l|l|l|l|l|l|l|l|l|}
\hline Data mining & 3 & 3 & 3 & 3 & 3 & 3 & 3 & 3 & 3 & 5 & 32 \\
\hline Ag & 3 & 3 & 3 & 1 & 1 & 3 & 3 & 3 & 3 & 5 & 28 \\
\hline
\end{tabular}

\begin{tabular}{|l|l|l|l|l|l|l|l|l|l|l|l|}
\hline Agentes Inteligentes & 3 & 3 & 3 & 1 & 1 & 3 & 3 & 3 & 3 & 5 & 28 \\
\hline Bus & & \\
\hline
\end{tabular}

\begin{tabular}{|l|l|l|l|l|l|l|l|l|l|l|l|}
\hline Business Intelligence & 3 & 3 & 3 & 5 & 3 & 3 & 3 & 3 & 3 & 5 & 34 \\
\hline Videocon
\end{tabular}

\begin{tabular}{|l|l|l|l|l|l|l|l|l|l|l|l|}
\hline Videoconferencia & 5 & 3 & 3 & 5 & 5 & 5 & 5 & 5 & 5 & 5 & 46 \\
\hline Acces Rem & 3 & 3 & 5 & 5 & 3 & 3 & 3 & 3 & 3 & 5 & 36 \\
\hline
\end{tabular}

\begin{tabular}{|l|l|l|l|l|l|l|l|l|l|l|l|}
\hline Acceso Remoto & 3 & 3 & 5 & 5 & 3 & 3 & 3 & 3 & 3 & 5 & 36 \\
\hline
\end{tabular}

\begin{tabular}{|l|l|l|l|l|l|l|l|l|l|l|l|}
\hline CRM & 3 & 3 & 3 & 3 & 3 & 3 & 3 & 3 & 3 & 5 & 32 \\
\hline
\end{tabular}

\begin{tabular}{|l|l|l|l|l|l|l|l|l|l|l|l|}
\hline Sistemas Expertos & 5 & 3 & 3 & 3 & 5 & 5 & 5 & 5 & 5 & 5 & 44 \\
\hline & 3 & 3 & 5 & 3 & 3 & 3 & 3 & 3 & 3 & 5 & 34 \\
\hline
\end{tabular}

\begin{tabular}{|l|l|l|l|l|l|l|l|l|l|l|l|}
\hline GPS & 3 & 3 & 5 & 3 & 3 & 3 & 3 & 3 & 3 & 5 & 34 \\
\hline BSC & 3 & 3 & 5 & 3 & 5 & 5 & 3 & 3 & 3 & 5 & 38 \\
\hline
\end{tabular}

\begin{tabular}{|l|l|l|l|l|l|l|l|l|l|l|l|l|}
\hline BSC & 3 & 3 & 5 & 3 & 5 & 5 & 3 & 3 & 3 & 5 & 38 \\
\hline
\end{tabular}

\begin{tabular}{|l|c|c|c|c|c|c|c|c|c|c|c|}
\hline Teletrabajo & 3 & 3 & 3 & 3 & 1 & 3 & 3 & 3 & 1 & 3 & 26 \\
\hline Cloud computing & 5 & 3 & 3 & 3 & 5 & 5 & 5 & 5 & 5 & 3 & 42 \\
\hline
\end{tabular}

\begin{tabular}{|l|l|l|l|l|l|l|l|l|l|l|l|}
\hline $\begin{array}{l}\text { Aplicaciones para } \\
\text { Ingeniería Mecánica }\end{array}$ & & & & & & & & & & & \\
\hline A & & & &
\end{tabular}

\begin{tabular}{|l|l|l|l|l|l|l|l|l|l|l|l|}
\hline $\begin{array}{l}\text { Autodesk AutoCAD } \\
\text { Plant 3D 2012 }\end{array}$ & 5 & 5 & 5 & 5 & 3 & 5 & 5 & 5 & 5 & 3 & 46 \\
\hline Aun
\end{tabular}

\begin{tabular}{|l|c|c|c|c|c|c|c|c|c|c|c|}
\hline $\begin{array}{l}\text { Autodesk }(\text { Inventor } \\
2012\end{array}$ & 5 & 5 & 5 & 5 & 3 & 5 & 5 & 5 & 5 & 3 & 46 \\
\hline
\end{tabular}

\begin{tabular}{l|l|l|l|l|l|l|l|l|l|l|l}
\hline $\begin{array}{l}\text { Ciemens software 3D } \\
\text { CAD Solid Edge }\end{array}$ & 5 & 5 & 5 & 5 & 3 & 5 & 5 & 5 & 5 & 3 & 46
\end{tabular}

\begin{tabular}{l|c|c|c|c|c|c|c|c|c|c|c|}
\hline AtinneSolidWorks & 5 & 5 & 5 & 5 & 3 & 5 & 5 & 5 & 5 & 3 & 46 \\
\hline Aun & & &
\end{tabular}

\begin{tabular}{|l|l|l|l|l|l|l|l|l|l|l|l|}
\hline Autodesk Factory & 5 & 5 & 5 & 5 & 3 & 5 & 5 & 5 & 5 & 3 & 46 \\
\hline Design Suite 2012
\end{tabular}

\begin{tabular}{l|l|l|l|l|l|l|l|l|l|l|l|}
\hline SolidWorks Flow & 5 & 5 & 5 & 5 & 3 & 5 & 5 & 5 & 5 & 3 & 46 \\
\hline
\end{tabular}

Simulation

AutoSoft Taller

Estándar 4.00

CADdy ++

\begin{tabular}{l|l|l|l|l|l|l|l|l|l|l|l}
\hline Mechanical Design & 5 & 5 & 5 & 5 & 3 & 5 & 5 & 5 & 5 & 3 & 46 \\
\hline
\end{tabular}

\begin{tabular}{|l|c|c|c|c|c|c|c|c|c|c|c|c|c|}
\hline Autodata 3.25 & 5 & 5 & 5 & 5 & 3 & 5 & 5 & 5 & 5 & 3 & 46 \\
\hline Mata & 5 & 5 & 5 & 5 & 3 & 5 & 5 & 5 & 5 & 3 & 46 \\
\hline
\end{tabular}

\begin{tabular}{|l|c|c|c|c|c|c|c|c|c|c|c|}
\hline Matlab R2010a & 5 & 5 & 5 & 5 & 3 & 5 & 5 & 5 & 5 & 3 & 46 \\
\hline Vial & 5 & 5 & 5 & 5 & 3 & 5 & 5 & 5 & 5 & 3 & 46 \\
\hline
\end{tabular}

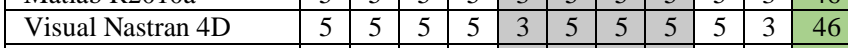

\begin{tabular}{|l|c|c|c|c|c|c|c|c|c|c|c|}
\hline ACIS & 5 & 5 & 5 & 5 & 3 & 5 & 5 & 5 & 5 & 3 & 46 \\
\hline Inven & 5 & 5 & 5 & 5 & 3 & 5 & 5 & 5 & 5 & 3 & 46
\end{tabular}

\begin{tabular}{|l|l|l|l|l|l|l|l|l|l|l|l|}
\hline $\begin{array}{l}\text { InventorCAM 3D y } \\
\text { 2D }\end{array}$ & 5 & 5 & 5 & 5 & 3 & 5 & 5 & 5 & 5 & 3 & 46 \\
\hline
\end{tabular}

ProENGINEER de

\begin{tabular}{l|l|l|l|l|l|l|l|l|l|l|l} 
PTC Parametric & 5 & 5 & 5 & 5 & 3 & 5 & 5 & 5 & 5 & 3 & 46
\end{tabular}

Technologies

\section{MPv9.0. Software \\ profesional para}

control y

administración del

mantenimiento

\begin{tabular}{|l|l|l|l|l|l|l|l|l|l|l|l|}
\hline MDESIGN (Diseño de & 5 & 5 & 5 & 5 & 3 & 5 & 5 & 5 & 5 & 3 & 46
\end{tabular}

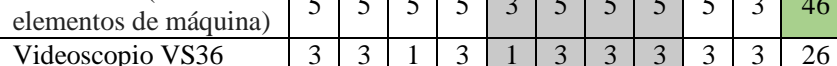

\begin{tabular}{|l|l|l|l|l|l|l|l|l|l|l|l|}
\hline $\begin{array}{l}\text { Equipo de Limpieza de } \\
\text { Inyectores Diesel }\end{array}$ & 3 & 1 & 1 & 3 & 1 & 3 & 1 & 3 & 1 & 3 & 20 \\
\hline A & & &
\end{tabular}

\begin{tabular}{|l|l|l|l|l|l|l|l|l|l|l|l|}
\hline $\begin{array}{l}\text { Adaptadores de } \\
\text { Limpieza }\end{array}$ & 3 & 3 & 1 & 3 & 1 & 3 & 5 & 3 & 3 & 3 & 28 \\
\hline
\end{tabular}

\begin{tabular}{|l|l|l|l|l|l|l|l|l|l|l|l|}
\hline Equipo Electrónico & 3 & 3 & 1 & 3 & 1 & 3 & 5 & 3 & 3 & 5 & 30 \\
\hline
\end{tabular}

\begin{tabular}{|llllllllllllll}
\hline $\begin{array}{l}\text { Laboratorio para } \\
\text { inyectores }\end{array}$ & 3 & 3 & 1 & 1 & 1 & 3 & 5 & 3 & 3 & 5 & 28 \\
\hline
\end{tabular}

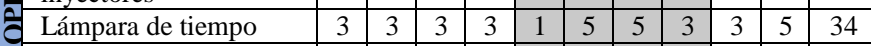

\begin{tabular}{|l|l|l|l|l|l|l|l|l|l|l|l|}
\hline Probador de sensores & 3 & 3 & 3 & 3 & 3 & 5 & 5 & 3 & 3 & 5 & 36 \\
\hline
\end{tabular}

\begin{tabular}{|l|l|l|l|l|l|l|l|l|l|l|l|}
\hline Pulsador de Inyectores & 3 & 3 & 5 & 3 & 3 & 5 & 5 & 3 & 3 & 5 & 38 \\
\hline Máquinas de Control & 5 & 5 & 5 & 5 & 3 & 5 & 5 & 5 & 5 & 3 & 46 \\
\hline
\end{tabular}

Numérico 


\begin{tabular}{|l|c|c|c|c|c|c|c|c|c|c|c|}
\hline $\begin{array}{l}\text { Maquinas de } \\
\text { Oleohidraulica y } \\
\text { Neumática }\end{array}$ & 5 & 5 & 5 & 5 & 3 & 5 & 5 & 5 & 5 & 3 & 46 \\
\hline Opel eléctrico 2013 & 1 & 1 & 1 & 1 & 3 & 5 & 1 & 1 & 1 & 3 & 18 \\
\hline Smart ForTwo 2012 & 1 & 1 & 1 & 1 & 3 & 3 & 1 & 1 & 1 & 3 & 16 \\
\hline $\begin{array}{l}\text { Nissan JukeKuro 2012 } \\
\text { Motor del Renault }\end{array}$ & 1 & 1 & 1 & 1 & 3 & 5 & 1 & 3 & 1 & 3 & 20 \\
\hline $\begin{array}{l}\text { Laguna 2012 } \\
\text { Lazda CX 5 }\end{array}$ & 1 & 1 & 1 & 1 & 3 & 3 & 1 & 1 & 1 & 3 & 16 \\
\hline $\begin{array}{l}\text { Mazda Con } \\
\text { Renault Koleos 2012 }\end{array} 1$ & 1 & 1 & 1 & 3 & 3 & 1 & 3 & 1 & 3 & 18 \\
\hline Motores eléctricos & 3 & 1 & 3 & 1 & 1 & 5 & 3 & 1 & 3 & 3 & 24 \\
\hline
\end{tabular}

\section{Matriz Tecnologías de Información vs Valores Estratégicos Deseados}

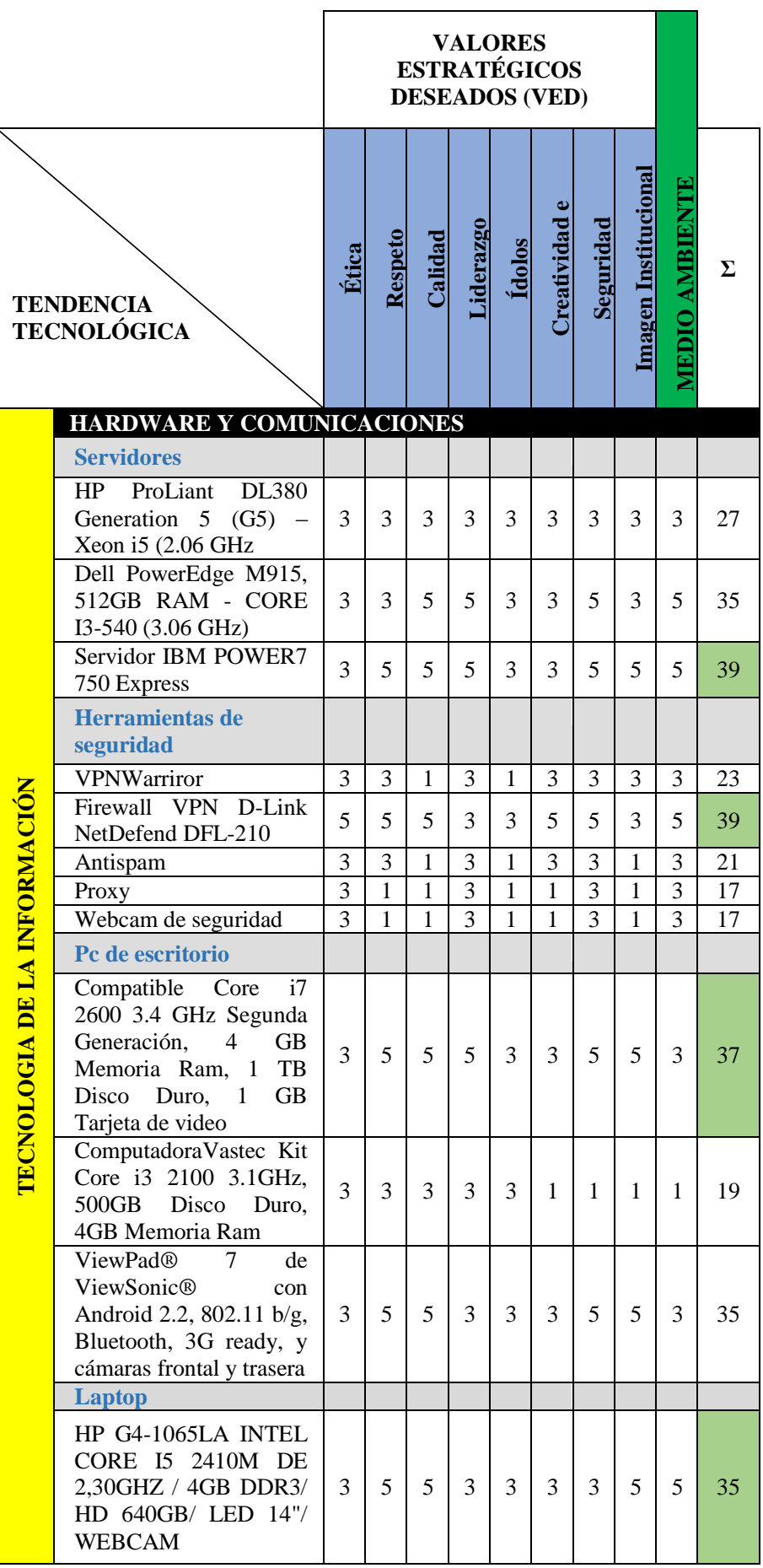

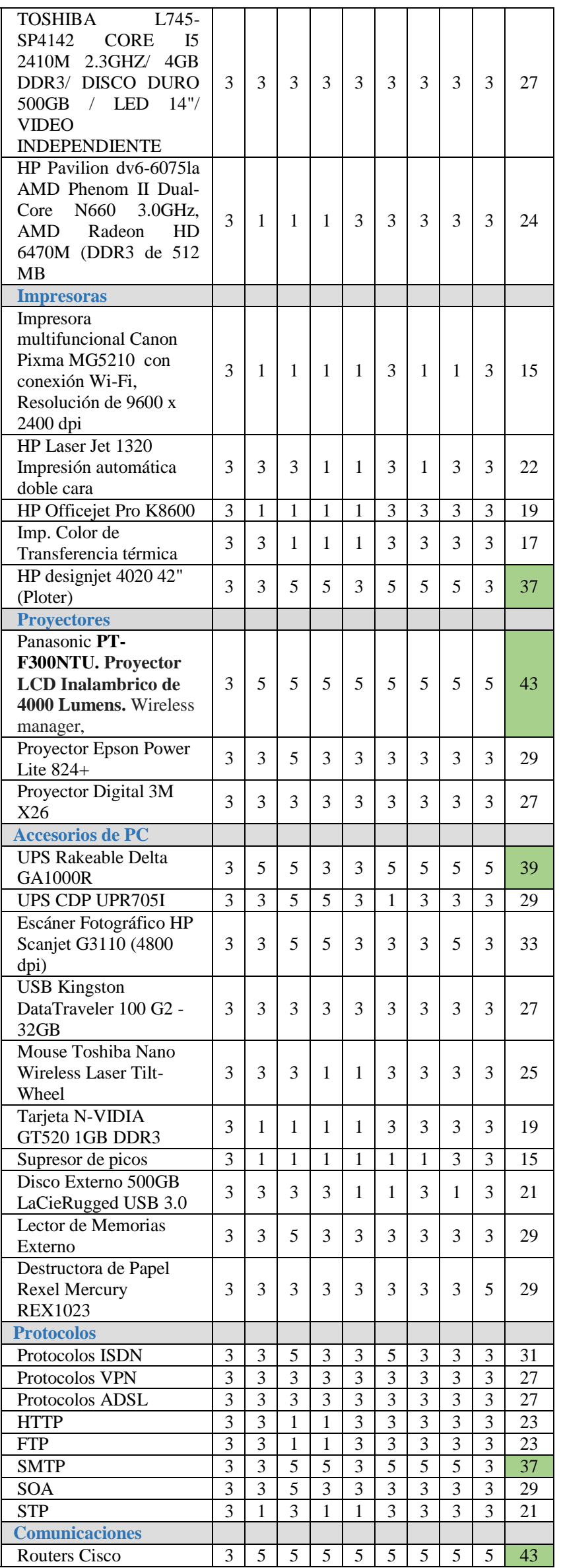




\begin{tabular}{|l|c|c|c|c|c|c|c|c|c|c|}
$\begin{array}{l}\text { Tarjetas red Wake On } \\
\text { LAN }\end{array}$ & 3 & 3 & 5 & 5 & 3 & 5 & 3 & 3 & 3 & 33 \\
\hline Switch 3Com & 3 & 3 & 5 & 5 & 5 & 5 & 5 & 5 & 5 & 41 \\
\hline Switch D-Link & 3 & 3 & 3 & 3 & 3 & 3 & 3 & 3 & 3 & 27 \\
\hline Access Point & 3 & 3 & 3 & 3 & 3 & 3 & 3 & 3 & 3 & 27 \\
\hline Estaciones LAN & 3 & 5 & 5 & 5 & 3 & 3 & 3 & 3 & 3 & 33 \\
\hline Central Telefónica & 3 & 3 & 3 & 3 & 3 & 3 & 3 & 3 & 3 & 27 \\
\hline $\begin{array}{l}\text { Teléfonos Corporativos } \\
\text { (Nextel,Claro, Movistar) }\end{array}$ & 3 & 3 & 3 & 3 & 3 & 3 & 3 & 3 & 3 & 27 \\
\hline Telefonía VoIP & 3 & 5 & 5 & 5 & 3 & 3 & 3 & 5 & 3 & 35 \\
\hline Tecnología Bluetooth & 3 & 3 & 3 & 3 & 3 & 3 & 3 & 3 & 3 & 27 \\
\hline $\begin{array}{l}\text { Tecnología Wifi- } \\
\text { Wimax }\end{array}$ & 3 & 3 & 5 & 3 & 3 & 5 & 3 & 3 & 3 & 31 \\
\hline
\end{tabular}

\section{SOFTWARE}

\begin{tabular}{|c|c|c|c|c|c|c|c|c|c|c|}
\hline Dperativos & & & & & & & & & & \\
\hline $\begin{array}{l}\text { MS Windows XP } \\
\text { Professional SP3 }\end{array}$ & 3 & 1 & 1 & 1 & 1 & 1 & 1 & 1 & 1 & 11 \\
\hline MS Windows 8 & 3 & 3 & 5 & 3 & 3 & 5 & 5 & 5 & 3 & 35 \\
\hline MS Windows 7 & 3 & 3 & 5 & 3 & 3 & 3 & 5 & 5 & 3 & 33 \\
\hline Windows Server 2008 & 3 & 5 & 5 & 5 & 3 & 5 & 5 & 5 & 3 & 39 \\
\hline Linux Red Hat & 3 & 3 & 3 & 3 & 3 & 3 & 3 & 3 & 3 & 27 \\
\hline Linux Ubuntu & 3 & 3 & 3 & 3 & 3 & 3 & 3 & 3 & 3 & 27 \\
\hline Linux Suse & 3 & 3 & 3 & 3 & 3 & 3 & 3 & 3 & 3 & 27 \\
\hline Android & 3 & 3 & 3 & 3 & 3 & 3 & 3 & 5 & 3 & 29 \\
\hline Base de Datos & & & & & & & & & & \\
\hline Oracle $11 \mathrm{G}$ & 3 & 5 & 5 & 5 & 3 & 5 & 5 & 5 & 3 & 39 \\
\hline MS Access 2010 & 3 & 3 & 1 & 1 & 1 & 1 & 1 & 1 & 3 & 15 \\
\hline DB2 & 3 & 5 & 5 & 3 & 3 & 5 & 5 & 5 & 3 & 37 \\
\hline MS SQL Server 2008 & 3 & 5 & 5 & 3 & 3 & 5 & 5 & 5 & 3 & 37 \\
\hline Postgree & 3 & 5 & 5 & 3 & 3 & 3 & 5 & 3 & 3 & 33 \\
\hline MySQL & 3 & 5 & 5 & 3 & 3 & 3 & 5 & 3 & 3 & 33 \\
\hline Ofimática & & & & & & & & & & \\
\hline Microsoft Office 2010 & 3 & 5 & 5 & 5 & 3 & 3 & 3 & 5 & 3 & 35 \\
\hline Open Office & 3 & 5 & 5 & 3 & 3 & 3 & 3 & 3 & 3 & 31 \\
\hline $\begin{array}{l}\text { Desarrollo de } \\
\text { aplicaciones de } \\
\text { escritorio }\end{array}$ & & & & & & & & & & \\
\hline Visual Studio 2012 & 3 & 3 & 5 & 5 & 3 & 5 & 5 & 3 & 3 & 35 \\
\hline VB\# & 3 & 3 & 5 & 3 & 3 & 3 & 5 & 3 & 3 & 31 \\
\hline Netbeans 7.0 & 3 & 3 & 5 & 3 & 3 & 5 & 5 & 3 & 3 & 33 \\
\hline $\begin{array}{l}\text { Desarrollo de } \\
\text { aplicaciones de Web }\end{array}$ & & & & & & & & & & \\
\hline Asp. Net & 3 & 5 & 5 & 3 & 3 & 5 & 5 & 5 & 3 & 37 \\
\hline JSP & 3 & 5 & 5 & 3 & 3 & 3 & 3 & 3 & 3 & 31 \\
\hline CGI & 3 & 5 & 5 & 3 & 3 & 3 & 3 & 3 & 3 & 31 \\
\hline XML & 3 & 5 & 5 & 3 & 3 & 3 & 3 & 5 & 3 & 33 \\
\hline JSF & 3 & 3 & 5 & 3 & 3 & 3 & 3 & 3 & 3 & 29 \\
\hline Struts & 3 & 1 & 5 & 3 & 3 & 3 & 3 & 3 & 3 & 27 \\
\hline $\begin{array}{l}\text { Desarrollo de } \\
\text { aplicaciones de } \\
\text { Móviles }\end{array}$ & & & & & & & & & & \\
\hline $\begin{array}{l}\text { J2ME Java Micro } \\
\text { Edition }\end{array}$ & 3 & 5 & 5 & 3 & 3 & 3 & 3 & 5 & 3 & 33 \\
\hline Android & 3 & 3 & 5 & 3 & 3 & 3 & 3 & 5 & 3 & 31 \\
\hline iTunes & 3 & 3 & 3 & 3 & 3 & 3 & 3 & 3 & 3 & 27 \\
\hline Windows Mobile & 3 & 5 & 5 & 3 & 3 & 5 & 3 & 5 & 3 & 35 \\
\hline Symbian & 3 & 3 & 3 & 3 & 3 & 3 & 3 & 3 & 3 & 27 \\
\hline Diseñadores & & & & & & & & & & \\
\hline Corel Draw X5 & 3 & 3 & 3 & 1 & 3 & 3 & 3 & 1 & 3 & 23 \\
\hline $\begin{array}{l}\text { Adobe Photoshop CS } \\
5.5\end{array}$ & 3 & 1 & 3 & 1 & 1 & 1 & 1 & 1 & 3 & 15 \\
\hline Adobe Ilustrator CS 5.5 & 3 & 3 & 5 & 3 & 3 & 3 & 3 & 3 & 3 & 31 \\
\hline Adobe Fireworks CS 5.5 & 3 & 1 & 3 & 1 & 1 & 1 & 1 & 3 & 3 & 17 \\
\hline Adobe Flash CS 5.5 & 3 & 3 & 3 & 1 & 3 & 1 & 3 & 1 & 3 & 21 \\
\hline $\begin{array}{l}\text { Adobe Dreamweaver } \\
\text { CS } 5.5\end{array}$ & 3 & 1 & 3 & 1 & 1 & 1 & 1 & 3 & 3 & 17 \\
\hline Utilitarios & & & & & & & & & & \\
\hline Internet Explorer & 3 & 1 & 1 & 3 & 1 & 3 & 3 & 3 & 3 & 21 \\
\hline
\end{tabular}

\begin{tabular}{|c|c|c|c|c|c|c|c|c|c|c|c|}
\hline & GoogleChrome & 3 & 1 & 1 & 3 & 1 & 1 & 3 & 3 & 3 & 19 \\
\hline & Google Apps & 3 & 3 & 3 & 3 & 3 & 5 & 3 & 5 & 3 & 31 \\
\hline & Mozilla & 3 & 5 & 5 & 3 & 3 & 5 & 5 & 3 & 3 & 35 \\
\hline & Antivirus Panda & 5 & 5 & 5 & 5 & 3 & 5 & 5 & 5 & 5 & 43 \\
\hline & Antivirus Symantec & 3 & 3 & 3 & 3 & 3 & 3 & 3 & 3 & 3 & 27 \\
\hline & Antivirus Avast & 3 & 3 & 3 & 3 & 3 & 3 & 3 & 3 & 3 & 27 \\
\hline & Adobe Reader 10.0 & 3 & 3 & 3 & 3 & \begin{tabular}{|l|l|l}
3 \\
\end{tabular} & 3 & 3 & 3 & 3 & 27 \\
\hline & Nero & 3 & 3 & 5 & 3 & 3 & 3 & 3 & 3 & 3 & 29 \\
\hline & Skype & 5 & 5 & 5 & 5 & 3 & 5 & 5 & 5 & 3 & 31 \\
\hline & $\begin{array}{l}\text { Aplicaciones } \\
\text { informáticas }\end{array}$ & & & & & & & & & & \\
\hline & Blended Learning & 3 & 5 & 5 & 5 & 3 & 3 & 3 & 3 & 3 & 33 \\
\hline & Data mining & 3 & 3 & 3 & 3 & 3 & 3 & 3 & 3 & 3 & 27 \\
\hline & Agentes Inteligentes & 3 & 5 & 5 & 5 & 3 & 5 & 3 & 5 & 3 & 37 \\
\hline & Business Intelligence & 3 & 3 & 3 & 3 & 3 & 3 & 3 & 3 & 3 & 27 \\
\hline & Videoconferencia & 3 & 5 & 5 & 5 & 3 & 3 & 5 & 5 & 5 & 39 \\
\hline & Acceso Remoto & 3 & 5 & 3 & 3 & 3 & 3 & 3 & 3 & 3 & 29 \\
\hline & CRM & 3 & 3 & 3 & 3 & 3 & 3 & 3 & 3 & 3 & 27 \\
\hline & Sistemas Expertos & 3 & 5 & 5 & 5 & 3 & 5 & 3 & 5 & 3 & 37 \\
\hline & GPS & 3 & 1 & 3 & 3 & 3 & 1 & 3 & 3 & 3 & 23 \\
\hline & BSC & 3 & 1 & 3 & 3 & 3 & 3 & 3 & 3 & 3 & 25 \\
\hline & Teletrabajo & 3 & 1 & 1 & 3 & 1 & 3 & 3 & 3 & 3 & 21 \\
\hline & Cloud computing & 3 & 5 & 5 & 5 & 3 & 5 & 3 & 3 & 3 & 35 \\
\hline & $\begin{array}{l}\text { Aplicaciones para } \\
\text { Ingeniería Mecánica }\end{array}$ & & & & & & & & & & \\
\hline & $\begin{array}{l}\text { Autodesk AutoCAD } \\
\text { Plant 3D 2012 }\end{array}$ & 3 & 5 & 5 & 5 & 5 & 5 & 5 & 5 & 3 & 41 \\
\hline & $\begin{array}{l}\text { Autodesk } ₫ \text { Inventor }{ }^{\circledR} \\
2012\end{array}$ & 3 & 5 & 5 & 5 & 5 & 5 & 5 & 5 & 3 & 41 \\
\hline & $\frac{\text { Siemens software 3D }}{\text { CAD Solid Edge }}$ & 3 & 5 & 5 & 5 & 5 & 5 & 5 & 5 & 3 & 41 \\
\hline & AtinneSolidWorks & 3 & 5 & 5 & 5 & 5 & 5 & 5 & 5 & 3 & 41 \\
\hline & $\begin{array}{l}\text { Autodesk Factory } \\
\text { Design Suite } 2012 \\
\end{array}$ & 3 & 5 & 5 & 5 & 5 & 5 & 5 & 5 & 3 & 41 \\
\hline & $\begin{array}{l}\text { SolidWorks Flow } \\
\text { Simulation }\end{array}$ & 3 & 5 & 5 & 5 & 5 & 5 & 5 & 5 & 3 & 41 \\
\hline & $\begin{array}{l}\text { AutoSoft Taller } \\
\text { Estándar } 4.00\end{array}$ & 3 & 5 & 5 & 5 & 5 & 5 & 5 & 5 & 3 & 41 \\
\hline & $\begin{array}{l}\text { CADdy ++ Mechanical } \\
\text { Design }\end{array}$ & 3 & 5 & 5 & 5 & 5 & 5 & 5 & 5 & 3 & 41 \\
\hline & Autodata 3.25 & 3 & 5 & 5 & 5 & 5 & 5 & 5 & 5 & 3 & 41 \\
\hline & Matlab R2010a & 3 & 5 & 5 & 5 & 5 & 5 & 5 & 5 & 3 & 41 \\
\hline & Visual Nastran 4D & 3 & 5 & 5 & 5 & 5 & 5 & 5 & 5 & 3 & 41 \\
\hline & ACIS & 3 & 5 & 5 & 5 & 5 & 5 & 5 & 5 & 3 & 41 \\
\hline & InventorCAM 3D y 2D & 3 & 5 & 5 & 5 & 5 & 5 & 5 & 5 & 3 & 41 \\
\hline & $\begin{array}{l}\text { ProENGINEER de PTC } \\
\text { Parametric } \\
\text { Technologies }\end{array}$ & 3 & 5 & 5 & 5 & 5 & 5 & 5 & 5 & 3 & 41 \\
\hline & $\begin{array}{l}\text { MPv9.0. Software } \\
\text { profesional para } \\
\text { control y } \\
\text { administración del } \\
\text { mantenimiento } \\
\end{array}$ & 3 & 5 & 5 & 5 & 5 & 5 & 5 & 5 & 3 & 41 \\
\hline & software & 3 & 5 & 5 & 5 & 5 & 5 & 5 & 5 & 3 & 41 \\
\hline & $\begin{array}{l}\text { MDESIGN (Diseño de } \\
\text { elementos de máquina) }\end{array}$ & 3 & 5 & 5 & 5 & 5 & 5 & 5 & 5 & 3 & 41 \\
\hline & Videoscopio VS36 & 3 & 3 & 3 & 3 & 1 & 3 & 3 & 3 & 3 & 25 \\
\hline & $\begin{array}{l}\text { Equipo de Limpieza de } \\
\text { Inyectores Diesel }\end{array}$ & 3 & 1 & 1 & 3 & 1 & 1 & 3 & 3 & 3 & 21 \\
\hline & $\begin{array}{l}\text { Adaptadores de } \\
\text { Limpieza }\end{array}$ & 3 & 1 & 1 & 3 & 1 & 1 & 3 & 3 & 3 & 21 \\
\hline כ & Equipo Electrónico & 1 & 1 & 3 & 1 & 1 & 1 & 3 & 3 & 3 & 17 \\
\hline$\underset{\frac{1}{x}}{\frac{1}{x}}$ & $\begin{array}{l}\text { Laboratorio para } \\
\text { inyectores }\end{array}$ & 1 & 3 & 3 & 3 & 1 & 3 & 3 & 3 & 3 & 23 \\
\hline & Lámpara de tiempo & 3 & 3 & 3 & 3 & 3 & 3 & 3 & 3 & 3 & 27 \\
\hline & Probador de sensores & 3 & 3 & 5 & 3 & 3 & 3 & 3 & 3 & 3 & 29 \\
\hline & Pulsador de Inyectores & 3 & 3 & 5 & 3 & 3 & 3 & 3 & 3 & 3 & 29 \\
\hline & $\begin{array}{l}\text { Máquinas de Control } \\
\text { Numérico }\end{array}$ & 3 & 5 & 5 & 5 & 3 & 5 & 5 & 5 & 5 & 41 \\
\hline
\end{tabular}




\begin{tabular}{|l|l|c|c|c|c|c|c|c|c|c|c|}
\hline & $\begin{array}{l}\text { Maquinas de } \\
\text { Oleohidraulica y } \\
\text { Neumática }\end{array}$ & 3 & 5 & 5 & 5 & 3 & 5 & 5 & 5 & 5 & 41 \\
\hline \multirow{3}{*}{$\underset{z}{*}$} & Opel eléctrico 2013 & 3 & 3 & 3 & 1 & 1 & 3 & 1 & 3 & 3 & 21 \\
\cline { 2 - 10 } & Smart ForTwo 2012 & 3 & 3 & 3 & 1 & 1 & 1 & 1 & 3 & 3 & 19 \\
\cline { 2 - 10 } & Nissan JukeKuro 2012 & 3 & 3 & 3 & 3 & 1 & 3 & 1 & 3 & 3 & 23 \\
\cline { 2 - 10 } & $\begin{array}{l}\text { Motor del Renault } \\
\text { Laguna 2012 }\end{array}$ & 3 & 1 & 1 & 1 & 1 & 3 & 1 & 3 & 3 & 17 \\
\cline { 2 - 11 } & Mazda CX 5 & 3 & 3 & 3 & 1 & 1 & 3 & 1 & 3 & 3 & 21 \\
\cline { 2 - 11 } & Renault Koleos 2012 & 3 & 1 & 1 & 1 & 1 & 3 & 1 & 3 & 3 & 17 \\
\cline { 2 - 10 } & Motores eléctricos & 3 & 3 & 3 & 3 & 3 & 3 & 3 & 3 & 3 & 27 \\
\hline
\end{tabular}

\begin{tabular}{|c|c|c|c|c|}
\hline \multicolumn{2}{|c|}{ TENDENCIA TECNOLÓGICA } & $\begin{array}{l}\Sigma \text { TI } \\
\text { VS } \\
\text { DOFA }\end{array}$ & $\begin{array}{l}\Sigma \text { TI } \\
\text { VS } \\
\text { VED }\end{array}$ & $\begin{array}{l}\text { ¿TOTA } \\
\quad \text { L }\end{array}$ \\
\hline \multirow{27}{*}{ 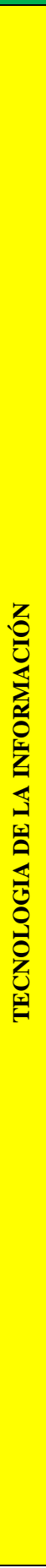 } & $\begin{array}{l}\text { HARDWARE Y } \\
\text { COMUNICACIONES }\end{array}$ & & & \\
\hline & Servidores & & & \\
\hline & $\begin{array}{l}\text { HP ProLiant DL380 Generation } 5 \\
\text { (G5) - Xeon i5 }(2.06 \mathrm{GHz}\end{array}$ & 28 & 27 & 55 \\
\hline & $\begin{array}{l}\text { Dell PowerEdge M915, 512GB } \\
\text { RAM - CORE I3-540 }(3.06 \mathrm{GHz})\end{array}$ & 32 & 35 & 67 \\
\hline & $\begin{array}{llll}\begin{array}{l}\text { Servidor } \\
\text { Express }\end{array} & \text { IBM } & \text { POWER7 } & 750 \\
\end{array}$ & 38 & 39 & 77 \\
\hline & Herramientas de seguridad & & & \\
\hline & VPNWarriror & 30 & 23 & 53 \\
\hline & $\begin{array}{l}\text { Firewall VPN D-Link NetDefend } \\
\text { DFL-210 }\end{array}$ & 35 & 39 & 74 \\
\hline & Antispam & 30 & 21 & 51 \\
\hline & Proxy & 28 & 17 & 45 \\
\hline & Webcam de seguridad & 32 & 17 & 49 \\
\hline & Pc de escritorio & & & \\
\hline & $\begin{array}{l}\text { Compatible Core i7 } 26003.4 \mathrm{GHz} \\
\text { Segunda Generación, } 4 \text { GB } \\
\text { Memoria Ram, } 1 \text { TB Disco Duro, } \\
1 \text { GB Tarjeta de video }\end{array}$ & 30 & 37 & 67 \\
\hline & $\begin{array}{l}\text { ComputadoraVastec Kit Core i3 } \\
2100 \text { 3.1GHz, 500GB Disco } \\
\text { Duro, 4GB Memoria Ram }\end{array}$ & 28 & 19 & 47 \\
\hline & $\begin{array}{l}\text { ViewPad } \circledast 7 \mathrm{de} \text { ViewSonic } \circledast \mathrm{con} \\
\text { Android } 2.2, \quad 802.11 \mathrm{~b} / \mathrm{g} \text {, } \\
\text { Bluetooth, } 3 \mathrm{G} \text { ready, y cámaras } \\
\text { frontal y trasera }\end{array}$ & 20 & 35 & 55 \\
\hline & Laptop & & & \\
\hline & $\begin{array}{l}\text { HP G4-1065LA INTEL CORE } \\
\text { I5 2410M DE 2,30GHZ / 4GB } \\
\text { DDR3/ HD 640GB/ LED 14"/ } \\
\text { WEBCAM }\end{array}$ & 30 & 35 & 65 \\
\hline & $\begin{array}{l}\text { TOSHIBA L745-SP4142 CORE } \\
\text { I5 2410M 2.3GHZ/ 4GB DDR3/ } \\
\text { DISCO DURO 500GB / LED } \\
\text { 14"/ VIDEO INDEPENDIENTE }\end{array}$ & 26 & 27 & 53 \\
\hline & $\begin{array}{ll}\text { HP Pavilion dv6-6075la } & \text { AMD } \\
\text { Phenom II Dual-Core N660 } \\
\text { 3.0GHz, AMD Radeon } \\
\text { 6470M (DDR3 de 512 MB } \\
\end{array}$ & 22 & 24 & 46 \\
\hline & Impresoras & & & \\
\hline & $\begin{array}{l}\text { Impresora multifuncional Canon } \\
\text { Pixma MG5210 con conexión } \\
\text { Wi-Fi, Resolución de } 9600 \text { x } \\
2400 \text { dpi }\end{array}$ & 24 & 15 & 39 \\
\hline & $\begin{array}{l}\text { HP Laser Jet } 1320 \text { Impresión } \\
\text { automática doble cara }\end{array}$ & 32 & 22 & 54 \\
\hline & HP Officejet Pro K8600 & 22 & 19 & 41 \\
\hline & $\begin{array}{l}\text { Imp. Color de Transferencia } \\
\text { térmica }\end{array}$ & 24 & 17 & 41 \\
\hline & HP designjet $402042 "$ (Ploter) & 50 & 37 & 87 \\
\hline & Proyectores & & & \\
\hline & $\begin{array}{lr}\text { Panasonic } & \text { PT- } \\
\text { F300NTUProyector } & \text { LCD } \\
\end{array}$ & 50 & 43 & 93 \\
\hline
\end{tabular}

\begin{tabular}{|c|c|c|c|}
\hline $\begin{array}{l}\text { Inalambrico de 4000 Lumens. } \\
\text { Wireless manager, Lámpara de } \\
5000 \text { horas y Filtro de } 10000 \\
\text { horas }\end{array}$ & & & \\
\hline $\begin{array}{l}\text { Proyector Epson Power Lite } \\
824+\end{array}$ & 35 & 29 & 64 \\
\hline Proyector Digital 3M X26 & 27 & 27 & 54 \\
\hline \multicolumn{4}{|l|}{ Accesorios de PC } \\
\hline UPS Rakeable Delta GA1000R & 26 & 39 & 65 \\
\hline UPS CDP UPR705I & 39 & 29 & 68 \\
\hline $\begin{array}{l}\text { Escáner Fotográfico HP Scanjet } \\
\text { G3110 (4800 dpi) }\end{array}$ & 26 & 33 & 59 \\
\hline $\begin{array}{l}\text { USB Kingston DataTraveler } 100 \\
\text { G2 - 32GB }\end{array}$ & 28 & 27 & 55 \\
\hline $\begin{array}{l}\text { Mouse Toshiba Nano Wireless } \\
\text { Laser Tilt-Wheel }\end{array}$ & 36 & 25 & 61 \\
\hline $\begin{array}{l}\text { Tarjeta N-VIDIA GT520 1GB } \\
\text { DDR3 }\end{array}$ & 28 & 19 & 47 \\
\hline Supresor de picos & 24 & 15 & 39 \\
\hline $\begin{array}{l}\text { Disco Externo 500GB } \\
\text { LaCieRugged USB 3.0 }\end{array}$ & 30 & 21 & 51 \\
\hline Lector de Memorias Externo & 34 & 29 & 63 \\
\hline $\begin{array}{l}\text { Destructora de Papel Rexel } \\
\text { Mercury REX1023 }\end{array}$ & 28 & 29 & 57 \\
\hline \multicolumn{4}{|l|}{ Protocolos } \\
\hline Protocolos ISDN & 34 & 31 & 65 \\
\hline Protocolos VPN & 32 & 27 & 59 \\
\hline Protocolos ADSL & 30 & 27 & 57 \\
\hline HTTP & 28 & 23 & 51 \\
\hline FTP & 28 & 23 & 51 \\
\hline SMTP & 32 & 37 & 59 \\
\hline SOA & 31 & 29 & 60 \\
\hline STP & 30 & 21 & 51 \\
\hline \multicolumn{4}{|l|}{ Comunicaciones } \\
\hline Routers Cisco & 32 & 43 & 75 \\
\hline Tarjetas red Wake On LAN & 30 & 33 & 63 \\
\hline Switch 3Com & 28 & 41 & 69 \\
\hline Switch D-Link & 26 & 27 & 53 \\
\hline Access Point & 36 & 27 & 63 \\
\hline Estaciones LAN & 24 & 33 & 57 \\
\hline Central Telefónica & 25 & 27 & 52 \\
\hline $\begin{array}{l}\text { Teléfonos Corporativos } \\
\text { (Nextel,Claro, Movistar) }\end{array}$ & 22 & 27 & 49 \\
\hline Telefonía VoIP & 20 & 35 & 55 \\
\hline Tecnología Bluetooth & 16 & 27 & 43 \\
\hline Tecnología Wifi-Wimax & 24 & 31 & 55 \\
\hline \multicolumn{4}{|l|}{ Sistemas Operativos } \\
\hline $\begin{array}{l}\text { MS Windows XP Professional } \\
\text { SP3 }\end{array}$ & 30 & 11 & 41 \\
\hline MS Windows 8 & 46 & 35 & 81 \\
\hline MS Windows 7 & 46 & 33 & 79 \\
\hline Windows Server 2008 & 40 & 39 & 79 \\
\hline Linux Red Hat & 30 & 27 & 57 \\
\hline Linux Ubuntu & 26 & 27 & 53 \\
\hline Linux Suse & 26 & 27 & 53 \\
\hline Android & 26 & 29 & 55 \\
\hline \multicolumn{4}{|l|}{ Base de Datos } \\
\hline Oracle $11 \mathrm{G}$ & 32 & 39 & 71 \\
\hline MS Access 2010 & 28 & 15 & 43 \\
\hline DB2 & 26 & 37 & 63 \\
\hline MS SQL Server 2008 & 42 & 37 & 79 \\
\hline Postgree & 22 & 33 & 55 \\
\hline MySQL & 24 & 33 & 57 \\
\hline \multicolumn{4}{|l|}{ Ofimática } \\
\hline Microsoft Office 2010 & 46 & 35 & 81 \\
\hline Open Office & 38 & 31 & 69 \\
\hline \multicolumn{4}{|l|}{$\begin{array}{l}\text { Desarrollo de aplicaciones de } \\
\text { escritorio }\end{array}$} \\
\hline Visual Studio 2012 & 40 & 35 & 75 \\
\hline VB\# & 36 & 31 & 67 \\
\hline
\end{tabular}




\begin{tabular}{|c|c|c|c|}
\hline Netbeans 7.0 & 40 & 33 & 77 \\
\hline \multicolumn{4}{|l|}{$\begin{array}{l}\text { Desarrollo de aplicaciones de } \\
\text { Web }\end{array}$} \\
\hline Asp. Net & 40 & 37 & 77 \\
\hline JSP & 36 & 31 & 67 \\
\hline CGI & 34 & 31 & 65 \\
\hline XML & 40 & 33 & 73 \\
\hline JSF & 40 & 29 & 69 \\
\hline Struts & 42 & 27 & 69 \\
\hline \multicolumn{4}{|l|}{$\begin{array}{l}\text { Desarrollo de aplicaciones de } \\
\text { Móviles }\end{array}$} \\
\hline J2ME Java Micro Edition & 42 & 33 & 75 \\
\hline Android & 40 & 31 & 71 \\
\hline iTunes & 38 & 27 & 65 \\
\hline Windows Mobile & 42 & 35 & 77 \\
\hline Symbian & 40 & 27 & 67 \\
\hline \multicolumn{4}{|l|}{ Diseñadores } \\
\hline Corel Draw X5 & 24 & 23 & 47 \\
\hline Adobe Photoshop CS 5.5 & 16 & 15 & 31 \\
\hline Adobe Ilustrator CS 5.5 & 30 & 31 & 61 \\
\hline Adobe Fireworks CS 5.5 & 16 & 17 & 33 \\
\hline Adobe Flash CS 5.5 & 18 & 21 & 39 \\
\hline Adobe Dreamweaver CS 5.5 & 18 & 17 & 35 \\
\hline \multicolumn{4}{|l|}{ Utilitarios } \\
\hline Internet Explorer & 44 & 21 & 65 \\
\hline GoogleChrome & 36 & 19 & 55 \\
\hline Google Apps & 30 & 31 & 61 \\
\hline Mozilla & 28 & 35 & 63 \\
\hline Antivirus Panda & 22 & 43 & 65 \\
\hline Antivirus Symantec & 31 & 27 & 58 \\
\hline Antivirus Avast & 24 & 27 & 51 \\
\hline Adobe Reader 10.0 & 18 & 27 & 45 \\
\hline Nero & 44 & 29 & 73 \\
\hline Skype & 36 & 31 & 67 \\
\hline \multicolumn{4}{|l|}{ Aplicaciones informáticas } \\
\hline Blended Learning & 36 & 33 & 69 \\
\hline Data mining & 32 & 27 & 59 \\
\hline Agentes Inteligentes & 28 & 37 & 65 \\
\hline Business Intelligence & 34 & 27 & 61 \\
\hline Videoconferencia & 46 & 39 & 85 \\
\hline Acceso Remoto & 36 & 29 & 65 \\
\hline CRM & 32 & 27 & 59 \\
\hline Sistemas Expertos & 44 & 37 & 81 \\
\hline GPS & 34 & 23 & 57 \\
\hline BSC & 38 & 25 & 63 \\
\hline Teletrabajo & 26 & 21 & 47 \\
\hline Cloud computing & 42 & 35 & 77 \\
\hline \multicolumn{4}{|l|}{$\begin{array}{l}\text { Aplicaciones para Ingeniería } \\
\text { Mecánica }\end{array}$} \\
\hline$\frac{\text { Autodesk AutoCAD Plant 3D }}{2012}$ & 46 & 41 & 87 \\
\hline Autodesk® Inventor® 2012 & 46 & 41 & 87 \\
\hline $\begin{array}{l}\text { Siemens software 3D CAD Solid } \\
\text { Edge }\end{array}$ & 46 & 41 & 87 \\
\hline AtinneSolidWorks & 46 & 41 & 87 \\
\hline $\begin{array}{l}\text { Autodesk Factory Design Suite } \\
2012\end{array}$ & 46 & 41 & 87 \\
\hline SolidWorks Flow Simulation & 46 & 41 & 87 \\
\hline AutoSoft Taller Estándar 4.00 & 46 & 41 & 87 \\
\hline CADdy ++ Mechanical Design & 46 & 41 & 87 \\
\hline Autodata 3.25 & 46 & 41 & 87 \\
\hline Matlab R2010a & 46 & 41 & 87 \\
\hline Visual Nastran 4D & 46 & 41 & 87 \\
\hline ACIS & 46 & 41 & 87 \\
\hline InventorCAM 3D y 2D & 46 & 41 & 87 \\
\hline $\begin{array}{l}\text { ProENGINEER de PTC } \\
\text { Parametric Technologies }\end{array}$ & 46 & 41 & 87 \\
\hline
\end{tabular}

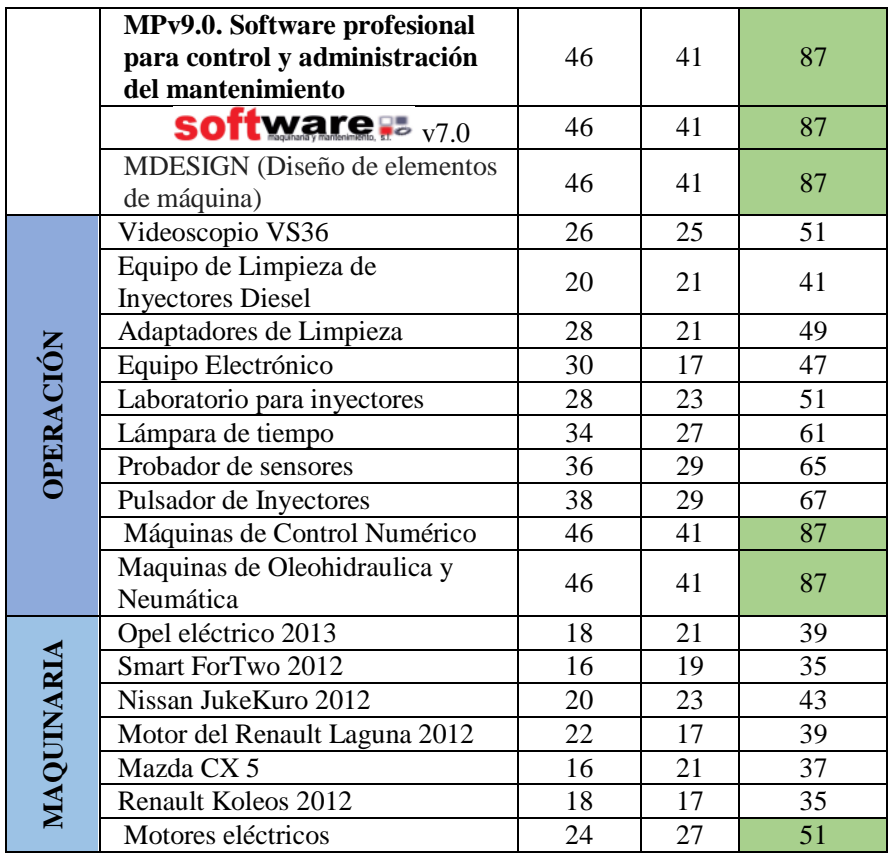

Matriz de Tiempo de Adquisición de las Tecnologías más Relevantes

\begin{tabular}{|c|c|c|}
\hline $\begin{array}{l}\text { TECNOLOGÍAS NECESARIAS } \\
\text { (Arquitectura de las TG) }\end{array}$ & $\begin{array}{l}\text { Es una } \\
\text { Tecnología } \\
\text { Actual }\end{array}$ & $\begin{array}{l}\text { Tiempo de } \\
\text { Adquisició } \\
\text { n en meses }\end{array}$ \\
\hline \multicolumn{3}{|l|}{ HARDWARE Y COMUNICACIONES } \\
\hline Servidor IBM POWER7 750 Express & $\begin{array}{l}\text { SI, Adquirir } \\
\text { equipo }\end{array}$ & 5 \\
\hline $\begin{array}{l}\text { Firewall VPN D-Link NetDefend DFL- } \\
210\end{array}$ & $\begin{array}{l}\text { SI, Adquirir } \\
\text { equipo }\end{array}$ & 2 \\
\hline $\begin{array}{l}\text { Compatible Core i7 } 26003.4 \mathrm{GHz} \\
\text { Segunda Generación, } 4 \text { GB Memoria Ram, } \\
1 \text { TB Disco Duro, } 1 \text { GB Tarjeta de video }\end{array}$ & $\begin{array}{l}\text { SI, Adquirir } \\
\text { equipo }\end{array}$ & 1 \\
\hline $\begin{array}{l}\text { HP G4-1065LA INTEL CORE I5 2410M } \\
\text { DE 2,30GHZ / 4GB DDR3/ HD 640GB/ } \\
\text { LED 14"/ WEBCAM }\end{array}$ & $\begin{array}{l}\text { SI, Adquirir } \\
\text { equipo }\end{array}$ & 1 \\
\hline $\begin{array}{l}\text { HP Laser Jet } 1320 \text { Impresión automática } \\
\text { doble cara }\end{array}$ & $\begin{array}{c}\text { SI, Adquirir } \\
\text { equipo }\end{array}$ & 1 \\
\hline HP designjet 4020 42" (Ploter) & $\begin{array}{l}\text { SI, Adquirir } \\
\text { equipo }\end{array}$ & 1 \\
\hline $\begin{array}{l}\text { Panasonic PT-LB3U 3200 Lúmenes de } \\
\text { brillo, Resolución 1024x768, Contraste } \\
\text { 600:1. }\end{array}$ & $\begin{array}{l}\text { SI, Adquirir } \\
\text { equipo }\end{array}$ & 2 \\
\hline UPS Rakeable Delta GA1000R & $\begin{array}{c}\text { SI, Adquirir } \\
\text { equipo }\end{array}$ & 1 \\
\hline $\begin{array}{l}\text { Escáner Fotográfico HP Scanjet G3110 } \\
(4800 \text { dpi) }\end{array}$ & $\begin{array}{l}\text { SI, Adquirir } \\
\text { equipo }\end{array}$ & 1 \\
\hline Protocolos ISDN & SI & - \\
\hline SMTP & SI & - \\
\hline Routers Cisco & $\begin{array}{l}\text { SI, Adquirir } \\
\text { equipo }\end{array}$ & 2 \\
\hline Switch 3Com & $\begin{array}{c}\text { SI, Adquirir } \\
\text { equipo }\end{array}$ & 2 \\
\hline Telefonía VoIP & SI & 3 \\
\hline Windows Server 2008 & $\begin{array}{l}\text { SI, Adquirir } \\
\text { licencia }\end{array}$ & 1 \\
\hline Oracle $11 \mathrm{G}$ & $\begin{array}{c}\text { SI, Adquirir } \\
\text { licencia }\end{array}$ & 1 \\
\hline MS SQL Server 2008 & $\begin{array}{c}\text { SI, Adquirir } \\
\text { licencia }\end{array}$ & 1 \\
\hline Microsoft Office 2010 & SI & - \\
\hline
\end{tabular}




\begin{tabular}{|c|c|c|}
\hline Visual Studio 2012 & SI & \\
\hline Asp. Net & SI & - \\
\hline J2ME Java Micro Edition & $\begin{array}{c}\text { SI, Adquirir } \\
\text { licencia }\end{array}$ & - \\
\hline Windows Mobile & $\begin{array}{l}\text { SI, Adquirir } \\
\text { licencia }\end{array}$ & 1 \\
\hline Adobe Ilustrator CS 5.5 & $\begin{array}{c}\text { SI, Adquirir } \\
\text { licencia }\end{array}$ & 1 \\
\hline Google Apps & SI & - \\
\hline Mozilla & SI & - \\
\hline Antivirus Panda & $\begin{array}{c}\text { SI, Adquirir } \\
\text { licencia }\end{array}$ & 1 \\
\hline Skype & SI & - \\
\hline Videoconferencia & $\begin{array}{c}\text { SI, Adquirir } \\
\text { equipo }\end{array}$ & 6 \\
\hline Sistemas Expertos & SI & - \\
\hline Cloud computing & SI & - \\
\hline Autodesk AutoCAD Plant 3D 2012 & $\begin{array}{l}\text { SI, Adquirir } \\
\text { licencia }\end{array}$ & 1 \\
\hline Autodesk® Inventor® 2012 & $\begin{array}{l}\text { SI, Adquirir } \\
\text { licencia }\end{array}$ & 1 \\
\hline Siemens software 3D CAD Solid Edge & $\begin{array}{c}\text { SI, Adquirir } \\
\text { licencia }\end{array}$ & 1 \\
\hline$\underline{\text { AtinneSolidWorks }}$ & $\begin{array}{c}\text { SI, Adquirir } \\
\text { licencia }\end{array}$ & 1 \\
\hline Autodesk Factory Design Suite 2012 & $\begin{array}{l}\text { SI, Adquirir } \\
\text { licencia }\end{array}$ & 1 \\
\hline$\underline{\text { SolidWorks Flow Simulation }}$ & $\begin{array}{l}\text { SI, Adquirir } \\
\text { licencia }\end{array}$ & 1 \\
\hline AutoSoft Taller Estándar 4.00 & $\begin{array}{l}\text { SI, Adquirir } \\
\text { licencia }\end{array}$ & 1 \\
\hline$\underline{\text { CADdy ++ Mechanical Design }}$ & $\begin{array}{l}\text { SI, Adquirir } \\
\text { licencia }\end{array}$ & 1 \\
\hline Autodata 3.25 & $\begin{array}{c}\text { SI, Adquirir } \\
\text { licencia }\end{array}$ & 1 \\
\hline Matlab R2010a & $\begin{array}{l}\text { SI, Adquirir } \\
\text { licencia }\end{array}$ & 1 \\
\hline Visual Nastran 4D & $\begin{array}{c}\text { SI, Adquirir } \\
\text { licencia }\end{array}$ & 1 \\
\hline ACIS & $\begin{array}{l}\text { SI, Adquirir } \\
\text { licencia }\end{array}$ & 1 \\
\hline InventorCAM 3D y $2 \mathrm{D}$ & $\begin{array}{l}\text { SI, Adquirir } \\
\text { licencia }\end{array}$ & 1 \\
\hline $\begin{array}{l}\text { ProENGINEER de PTC Parametric } \\
\text { Technologies }\end{array}$ & $\begin{array}{l}\text { SI, Adquirir } \\
\text { licencia }\end{array}$ & 1 \\
\hline $\begin{array}{l}\text { MPv9.0. Software profesional para } \\
\text { control y administración del } \\
\text { mantenimiento }\end{array}$ & $\begin{array}{l}\text { SI, Adquirir } \\
\text { licencia }\end{array}$ & 1 \\
\hline 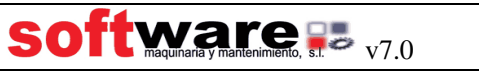 & $\begin{array}{c}\text { SI, Adquirir } \\
\text { licencia }\end{array}$ & 1 \\
\hline $\begin{array}{l}\text { MDESIGN (Diseño de elementos de } \\
\text { máquina) }\end{array}$ & $\begin{array}{l}\text { SI, Adquirir } \\
\text { licencia }\end{array}$ & 1 \\
\hline \multicolumn{3}{|l|}{ TECNOLOGÍAS DE OPERACIONES } \\
\hline Máquinas de Control Numérico & $\begin{array}{l}\text { SI, Adquirir } \\
\text { máquinas }\end{array}$ & 6 \\
\hline Maquinas de Oleohidraulica y Neumática & $\begin{array}{l}\text { SI, Adquirir } \\
\text { máquinas }\end{array}$ & 6 \\
\hline Motores eléctricos & $\begin{array}{l}\text { SI, Adquirir } \\
\text { máquinas }\end{array}$ & 6 \\
\hline
\end{tabular}

\section{Arquitectura de las tecnologías de información} Arquitectura de la intranet

La intranet a implementar tendrá la siguiente arquitectura:

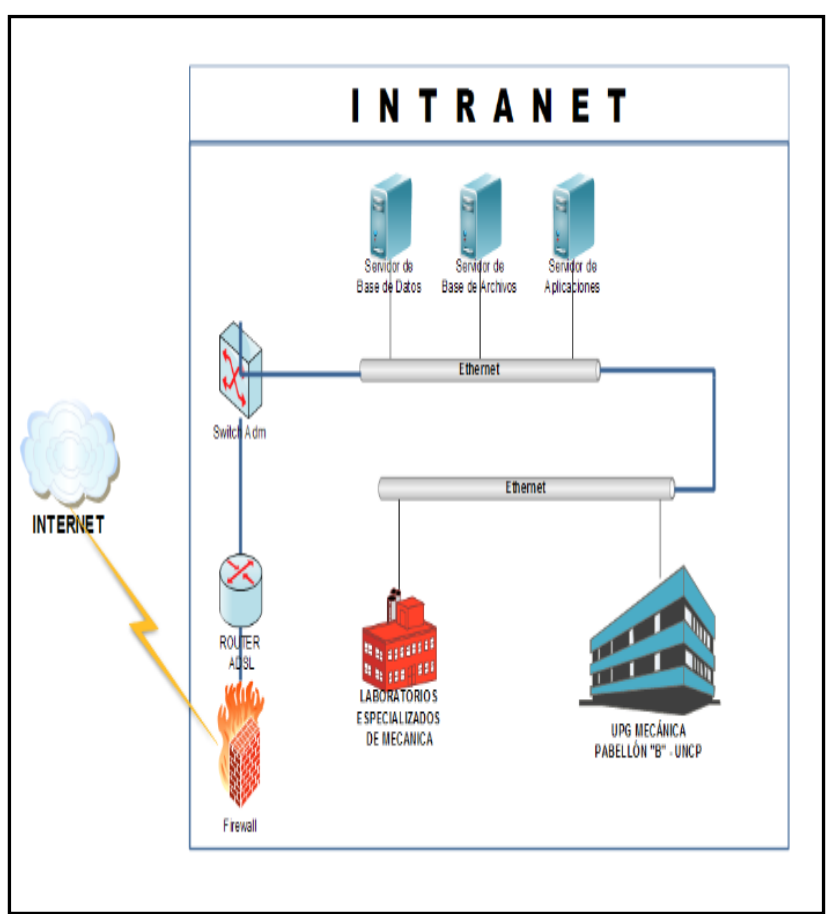

Figura: Modelo General de la Arquitectura de Intranet

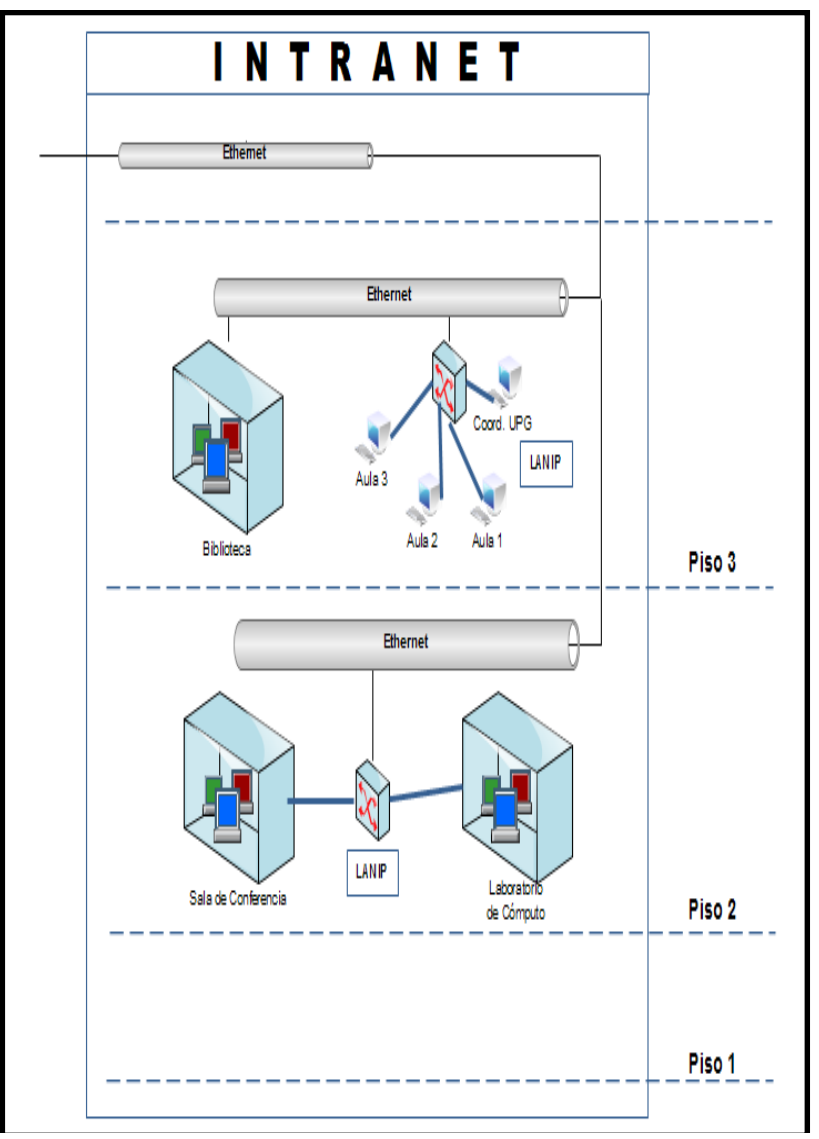

Figura: Arquitectura de Intranet en el Pabellón "B"

\section{Arquitectura de la extranet}

Para la implementación de la extranet, se consideró las relaciones de la UPG de Ing. Mecánica con sus Stakeholders: 


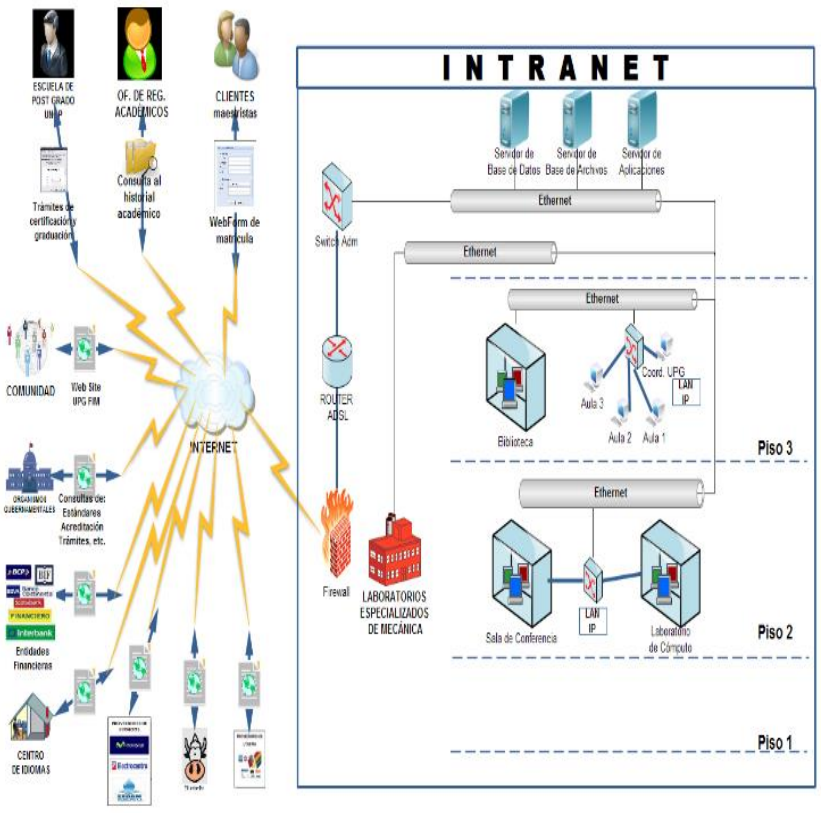

Sistemas de información estratégicos

* Sistemas de información de investigación

Este sistema permitirá la administración de las investigaciones que se realizan en la Unidad de Posgrado, esto es automatizará todo el proceso de investigación que realiza el maestristas tales como:

Registrar la inscripción de los proyectos de investigación', incluyendo datos del autor y asesor. Registrar la designación de los revisores, incluyendo plazos y fechas de presentación de informes, observaciones, subsanaciones a informes.

Registrar las solicitudes de designación de hora y fecha: designación de jurados, hora y fecha de sustentación, acta de sustentación, resultados de la sustentación.

Registrar las actividades de graduación: solicitud de diploma, actas de aprobación de la sustentación, fecha de expedición, etc.

El sistema permitirá obtener información estratégica de las investigaciones que se realizan, tales como:

Numero de investigaciones realizadas por periodo. Numero de investigaciones realizadas por área de investigación.

Número de investigaciones sustentadas y aprobadas o desaprobadas.

Número de investigaciones asesoradas por un cierto docente.

\section{* Sistemas de información académica}

Este sistema permitirá la administración académica de la UPG, referente a matrículas y notas, por lo que automatizará todas las actividades referidas a los procesos mencionados, tales como:

Registrar las matrículas de los maestristas vía internet.
Registrar la distribución horaria por docente, ciclo y mención para que el alumno pueda matricularse. Registrar el ingreso de notas vía web, etc.

El sistema permitirá obtener información estratégica de las investigaciones que se realizan, tales como:

Emitir constancia de matrículas a los alumnos.

Informar número de alumnos matriculados, por curso, ciclo y mención

Emitir horarios de clases por curso, ciclo, docente y mención.

Emitir boletas de notas.

Emitir registros.

Emitir reportes estadísticos del número de alumnos matriculados por periodo.

Emitir reportes estadísticos del número de alumnos aprobados y desaprobados por curso, ciclo, mención y periodo, etc.

\section{DISCUSIÓN}

Como se pudo apreciar se ha realizado la propuesta de las tecnologías que debe adoptar la Unidad de Posgrado de la Facultad de Ing. Mecánica de la UNCP a fin de lograr ventaja competitiva; esto a razón de que "en la actualidad vivimos en un entorno empresarial cada vez más interesado en la adquisición de nuevas tecnologías de la información y la comunicación (TICs), toda vez que están son consideradas como parte de del planeamiento estratégico institucional, y su adopción e implementación generará una ventaja competitiva sobre su sector" (Sáenz, 2018).

Bajo este contexto es primordial establecer los costos en el que debe incurrir la UPG de la Facultad de Ing. Mecánica, para la adopción e implementación de las TIC.

Resumen de costos del servicio
\begin{tabular}{|l|l|}
\hline \multicolumn{2}{|c|}{ Resumen de Costos de la Solución } \\
\hline & $\$$ \\
Costo Inicial de la Solución & $12,398.00$ \\
\hline & $\$$ \\
Costo de Administración & $4,950.00$ \\
\hline & $\$$ \\
Costo de Operación & $1,200.00$ \\
\hline & $\$$ \\
Costo de Soporte & $1,200.00$ \\
\hline
\end{tabular}

\begin{tabular}{|lc|}
\hline & $\$$ \\
Total de la Solución & $19,748.00$ \\
\hline
\end{tabular}

\begin{tabular}{|c|c|}
\hline $\begin{array}{l}\text { Total de la Solución en } \\
\text { Soles }\end{array}$ & $\begin{array}{l}\text { S/ } \\
54,899.44\end{array}$ \\
\hline
\end{tabular}

\section{CONCLUSIONES}

1. La implementación del Plan Estratégico de Tecnología de Información (PETI) en la UPG de la Facultad de Ingeniería Mecánica de la UNCP, implica cambios significativos en las estrategias de negocio tanto a nivel operativo y de Tecnologías de Información (TI), los mismo que se materializan con la implementación de laboratorios especializados de mecánica que incluye el uso de simuladores, renovación de laboratorios de 
cómputo, implementación de nuevos sistemas de información, rediseño de los procesos estratégicos y operativos. Aspectos estos que permitirán a la organización una gestión estratégica de la información.

2. La planificación de la Arquitectura de Sistemas realizado para la UPG de la Facultad de Ingeniería Mecánica de la UNCP proporciona un soporte a la implementación de la Arquitectura de Información de la UPG.

3. Los cambios propuestos son factibles como consecuencia de la definición de la estructura de la organización y el manejo de los recursos humanos en TI (hardware, software, comunicaciones).

4. En toda organización como la UPG la información es un valor que se transforma en un factor crítico de éxito hoy en día, pues gracias a ella se pueden tomar decisiones que van a repercutir en las diversas unidades y departamentos para apoyar la estrategia de la organización. El PETI se encarga de administrar eficientemente el manejo de la información garantizando la concordancia de la estrategia de la institución y la estrategia de TI dándonos una ventaja competitiva.

\section{AGRADECIMIENTOS}

Nuestros agradecimientos a las autoridades, personal docente y no docente de la Facultad de Ingeniería Mecánica de la UNCP.

\section{LITERATIURA CITADA}

En orden alfabético.

Abramowics, W., \& Mayr, H. (2007). Technologies for Business Information Systems. Dordrecht Holanda: Springer Editors.

CCTI, G. (2018). DEfinición de PETI. Obtenido de https://ccti.com.co/index.php/definicion-peti

Esteves, A. (03 de noviembre de 2012). La Metodología PETI. Obtenido de http://metodologiapetiensayo1.blogspot.com/

Hernandez, R., Fernándes , C., \& Baptista, P. (2007).
Fundamentos de metodología de investigación. Madrid: McGraw Hill.

Herrscher, E. (2010). El valor sistémico de las organizaciones. Buenos Aires: Granica Editores.

Maxwell, J. (2002). Liderazgo Eficaz. Estados Unidos de América: Editores Vida.

Oficina Técnica de Informática INEI. (2017). Plan Estratégico de Tecnologías de la Información (PETI) del Instituto Nacional de Estadística $e$ Informática - INEI. Obtenido de https://www.inei.gob.pe/media/pte/PETI/Plan_Est rategico_de_Tecnologia_de_la_InformacionPETI\%20VF.PDF

Sáenz, C. (18 de febrero de 2018). Cuál es el verdadero valor de las tecnologías de la información. Obtenido de https://www.esan.edu.pe/conexion/actualidad/201 8/02/19/tecnologias-de-la-informacionanalizando-el-valor-estrategico-de-unaherramienta/

Sub Jefatura de Tecnologías de Información-SERVIR. (2016). PLAN ESTRATÉGICO DE TECNOLOGÍAS DE INFORMACIÓN DE SERVIR (PETI 2016-2021). Obtenido de https://storage.servir.gob.pe/transparenciapeti/PETI-2016.pdf

Ulrich, D., \& Smallwood, N. (2010). Nuestro recorrido de liderazgo. Estados Unidos de América: Norma.

Ulrich, D., Zenger, J., \& Smallwood, N. (1999). Liderazgo basado en resultados. Bogota: Norma.

UNCP, U. (01 de 01 de 1999). Reseña Histórica. Obtenido de http://www.uncp.edu.pe/?q=historia

Warren, B., \& Nanus, B. (1985). Lideres: las cuatro claves del liderazgo eficaz. Barcelona: Norma.

Wordpress, A. (setiembre de 2016). Planeación Estratégica de Tecnologías de Información (PETI). Obtenido de https://ale170.wordpress.com/2013/09/16/planeac ion-estrategica-de-tecnologias-de-informacionpeti 